\title{
A new similarity measure for nonlocal filtering in the presence of multiplicative noise
}

\author{
T. Teuber * and A. Lang ${ }^{\dagger}$
}

February 9, 2011

\begin{abstract}
This paper presents a new similarity measure and nonlocal filters for images corrupted by multiplicative noise. The considered filters are generalizations of the nonlocal means filter of Buades et al., which is known to be well suited for removing additive Gaussian noise. To adapt to different noise models, the patch comparison involved in this filter has first of all to be performed by a suitable noise dependent similarity measure. To this purpose, we start by studying a probabilistic measure recently proposed for general noise models by Deledalle et al. We analyze this measure in the context of conditional density functions and examine its properties for images corrupted by additive and multiplicative noise. Since it turns out to have unfavorable properties for multiplicative noise we deduce a new similarity measure consisting of a probability density function specially chosen for this type of noise. The properties of our new measure are studied theoretically as well as by numerical experiments. To obtain the final nonlocal filters we apply a weighted maximum likelihood estimation framework, which also incorporates the noise statistics. Moreover, we define the weights occurring in these filters using our new similarity measure and propose different adaptations to further improve the results. Finally, restoration results for images corrupted by multiplicative Gamma and Rayleigh noise are presented to demonstrate the very good performance of our nonlocal filters.
\end{abstract}

\section{Introduction}

In 2005, Buades et al. proposed the nonlocal (NL) means filter [3], which is based on the following idea: Each pixel $f_{i}$ of a given noisy image $f$ is compared together with its neighboring pixels to other image patches. For each comparison a weight is assigned depending on the similarity of the image patches. The restored pixel $\widetilde{u}_{i}$ is now the weighted average of the central pixels of these patches using the obtained weights. For a discrete image $f \in \mathbb{R}^{m, n}$, $N=m n$, we have in detail

$$
\widetilde{u}_{i}=\frac{1}{C_{i}} \sum_{j=1}^{N} w_{N L}(i, j) f_{j}
$$

\footnotetext{
${ }^{*}$ University of Kaiserslautern, Dept. of Mathematics, Erwin-Schrödinger-Straße, 67663 Kaiserslautern, Germany (tteuber@mathematik.uni-kl.de)

${ }^{\dagger}$ ETH Zürich, Seminar for Applied Mathematics, Rämistr. 101, 8092 Zürich, Switzerland (annika.lang@sam.math.ethz.ch)
} 
with $C_{i}:=\sum_{j=1}^{N} w_{N L}(i, j)$. If the image patches with centers $f_{i}, f_{j}$ are given by $f_{i+I}$, resp. $f_{j+I}$ for $I$ denoting an appropriate index set, then the weights are obtained by

$$
w_{N L}(i, j)=\exp \left(-\frac{1}{h} \sum_{k \in I} g_{a, k}\left|f_{i+k}-f_{j+k}\right|^{2}\right) .
$$

Here, the parameter $h>0$ is used to control the amount of filtering. The vector $g_{a}=\left(g_{a, k}\right)_{k \in I}$ represents usually a sampled two dimensional Gaussian kernel with mean zero and standard deviation $a$ steering the influence of neighboring pixels on the weight.

In the past five years this filter has been extensively studied and further improved in various directions. An analysis and comparison with other state-of-the-art image denoising methods as well as an overview of recent developments in this area can for example be found in [4].

Among other improvements, several authors proposed different approaches to adapt the nonlocal means filter to noise statistics. Kervrann et al. proposed the so-called Bayesian NL means filter [18] which gave a first possibility to incorporate the statistics of the noise. In [5] this filter has been applied to remove speckle noise in ultrasound images. An approach for Rician noise was presented in [25]. Another generalization of the original NL means filter in a probabilistic framework was given by Deledalle et al. in [7]. Here, a central step was to incorporate the noise statistics in a suitable way into the weight definition of their filters. To illustrate the basic idea, let us rewrite the weights of the NL means filter in the form

$$
w_{N L}(i, j)=\prod_{k \in I} s_{N L}\left(f_{i+k}, f_{j+k}\right)^{\frac{g_{a, k}}{h}} \text { with } s_{N L}(x, y):=\exp \left(-|x-y|^{2}\right) .
$$

Hence, the weights can be constructed by taking the product over $s_{N L}\left(f_{i+k}, f_{j+k}\right)^{\frac{g_{a, k}}{h}}$ for all pairs of pixels $f_{i+k}$ and $f_{j+k}$ of the two image patches. The function $s_{N L}: \mathbb{R} \times \mathbb{R} \rightarrow(0,1]$ can be viewed as a similarity measure, where $s_{N L}\left(f_{i+k}, f_{j+k}\right)$ is supposed to be close to one if the original noise free pixels belonging to $f_{i+k}$ and $f_{j+k}$ have been the same and it should be close to zero if not. For images corrupted by additive Gaussian noise, $s_{N L}$ is known to perform well. Unfortunately, it can be far from optimal for other types of noise. Hence, the challenge is to find a suitable noise adapted similarity measure, which can cope with different types of noise. The similarity measure proposed for general noise models in [7] was demonstrated to perform well for images corrupted by additive Gaussian noise, noise following a Nakagami-Rayleigh distribution and Poisson noise studied in [8].

The aim of this paper is to present a new similarity measure specially designed for comparing data in the presence of multiplicative noise. This type of noise occurs in real life applications such as for example ultrasound and synthetic aperture radar (SAR) imaging. In contrast to recent image restoration methods, see, e.g., [1, 9, 10, 24], we use our new measure to define suitable nonlocal filters for removing multiplicative noise in images.

To start with, we revisit the similarity measure proposed in [7] in Section 2 and analyze it in the framework of conditional density functions. Moreover, we study its properties for images corrupted by additive and multiplicative noise. Since it turns out to be well suited for additive noise, but to have unfavorable properties for multiplicative noise, we deduce our new measure given by a noise dependent density function in Section 3. The advantages of this measure are shown theoretically as well as by different examples and experiments. Next, we deduce 
our nonlocal filters by maximum likelihood estimation in Section 4 and define the involved weights using our new similarity measure. Moreover, we present different modifications to further improve the results. The very good performance of our new filters is demonstrated for images corrupted by multiplicative Gamma and Rayleigh noise in Section 5 and finally, we end with conclusions in Section 6.

\section{The similarity measure of Deledalle et al.}

To start with, we revisit the similarity measure applied by Deledalle, Denis and Tupin in [7]. To fix the notation, all random variables are named with capital letters and are supposed to be real-valued, continuous and defined on a fixed probability space $(\Omega, \mathcal{F}, P)$. Moreover, $p_{X}$ stands for the density of the random variable $X$. For any $x$ with $p_{X}(x)>0$, the conditional density of a random variable $Y$ given $X=x$, is defined by

$$
p_{Y \mid X}(\cdot \mid x):=\frac{p_{Y, X}(\cdot, x)}{p_{X}(x)}
$$

see, e.g., [15, p. 104]. It holds that

$$
\int_{-\infty}^{y} p_{Y \mid X}(t \mid x) d t=\lim _{\varepsilon \rightarrow 0^{+}} P(Y \leq y \mid X \in(x-\varepsilon, x+\varepsilon]),
$$

which shows the connections between a conditional density and the corresponding conditional probability. Note that Appendix A contains a collection of results from probability theory, which will be used in this and the subsequent sections.

In the following, we suppose that the noisy pixels $f_{i}$ are realizations of independent continuous random variables $F_{i}$ and the corresponding original noise free pixels $u_{i}$ are realizations of the independent identically distributed random variables $U_{i}, i=1, \ldots, N$. Moreover, let all $f_{i}$ be contaminated by the same noise model with equal parameters. Since for the following considerations we need only two pixels, we set $N=2$ for simplicity.

Now, to measure whether $u_{1}=u_{2}$ by the noisy observations $f_{1}, f_{2}$, Deledalle et al. suggest to use a so-called 'similarity probability' denoted by $p\left(\theta_{1}=\theta_{2} \mid f_{1}, f_{2}\right)$. In their paper, $\theta_{i}$ is a parameter depending deterministically on $u_{i}$ and we consider $\theta_{i}=u_{i}, i=1,2$. Since in general it is not clear what the probability or even conditional density function of $U_{1}=U_{2}$ given $F_{1}=f_{1}, F_{2}=f_{2}$ is, see, e.g., [15, p. 111], we start by interpreting the 'similarity probability' as a conditional density: In [7] it is set to be

$$
p\left(u_{1}=u_{2} \mid f_{1}, f_{2}\right):=\int_{S} p_{U_{1} \mid F_{1}}\left(u \mid f_{1}\right) p_{U_{2} \mid F_{2}}\left(u \mid f_{2}\right) d u,
$$

where we need to have $p_{F_{i}}\left(f_{i}\right)>0, i=1,2$ and define $S:=\operatorname{supp}\left(p_{U_{i}}\right)$. By the definition of the conditional density it holds that

$$
\int_{S} p_{U_{1} \mid F_{1}}\left(u \mid f_{1}\right) p_{U_{2} \mid F_{2}}\left(u \mid f_{2}\right) d u=\frac{\int_{S} p_{U_{1}, F_{1}}\left(u, f_{1}\right) p_{U_{2}, F_{2}}\left(u, f_{2}\right) d u}{p_{F_{1}}\left(f_{1}\right) p_{F_{2}}\left(f_{2}\right)} .
$$


Furthermore, we obtain by Theorem A.1 and the independence of $\left(U_{1}, F_{1}\right)$ and $\left(U_{2}, F_{2}\right)$ that

$$
p_{U_{1}-U_{2}, U_{2}, F_{1}, F_{2}}\left(x, u, f_{1}, f_{2}\right)=p_{U_{1}, F_{1}}\left(x+u, f_{1}\right) p_{U_{2}, F_{2}}\left(u, f_{2}\right)
$$

and thus,

$p_{U_{1}-U_{2}, F_{1}, F_{2}}\left(0, f_{1}, f_{2}\right)=\int_{-\infty}^{\infty} p_{U_{1}-U_{2}, U_{2}, F_{1}, F_{2}}\left(0, u, f_{1}, f_{2}\right) d u=\int_{S} p_{U_{1}, F_{1}}\left(u, f_{1}\right) p_{U_{2}, F_{2}}\left(u, f_{2}\right) d u$.

Inserting this in (4) shows with (3) and the independence of $F_{1}, F_{2}$ that

$$
p\left(u_{1}=u_{2} \mid f_{1}, f_{2}\right)=p_{U_{1}-U_{2} \mid\left(F_{1}, F_{2}\right)}\left(0 \mid f_{1}, f_{2}\right) .
$$

For this reason, we will in the following refer to the 'similarity probability' by the conditional density on the right hand side. By (4) it can also be expressed in the form

$$
p_{U_{1}-U_{2} \mid\left(F_{1}, F_{2}\right)}\left(0 \mid f_{1}, f_{2}\right)=\frac{\int_{S} p_{U_{1}}(u) p_{U_{2}}(u) p_{F_{1} \mid U_{1}}\left(f_{1} \mid u\right) p_{F_{2} \mid U_{2}}\left(f_{2} \mid u\right) d u}{p_{F_{1}}\left(f_{1}\right) p_{F_{2}}\left(f_{2}\right)} .
$$

Since in general no knowledge about the distribution of the random variables $U_{i}$ is given, Deledalle et al. propose to neglect the densities $p_{U_{i}}$ and $p_{F_{i}}, i=1,2$ and to consider only

$$
s_{D D T}\left(f_{1}, f_{2}\right):=\int_{S} p_{F_{1} \mid U_{1}}\left(f_{1} \mid u\right) p_{F_{2} \mid U_{2}}\left(f_{2} \mid u\right) d u .
$$

This measure is very close to the one investigated for block matching in [19]. One may ask if $s_{D D T}$ can also be interpreted in terms of a conditional density function similar to (5). For the case of additive noise with $S=\mathbb{R}$ the answer is yes as we will see in the next subsection.

\subsection{Properties in the presence of additive noise}

In the following, suppose additionally that $V_{i}, i=1,2$, are independent identically distributed random variables, which follow some noise distribution. Moreover, let $u_{i}$ be corrupted by additive noise, i.e. $f_{i}:=u_{i}+v_{i}$ and

$$
F_{i}:=U_{i}+V_{i}, \quad i=1,2,
$$

where each $v_{i}$ is a realization of the random variable $V_{i}$. Consider further all $U_{i}, V_{i}, i=1,2$ to be pairwise independent. Under these conditions, we can show that $s_{D D T}$ has the following properties:

Proposition 2.1. For the described additive noise model with $S=\operatorname{supp}\left(p_{U_{i}}\right)=\mathbb{R}$ we have

$$
s_{D D T}\left(f_{1}, f_{2}\right)=p_{V_{1}-V_{2}}\left(f_{1}-f_{2}\right)=p_{F_{1}-F_{2} \mid U_{1}-U_{2}}\left(f_{1}-f_{2} \mid 0\right) \quad \forall f_{1}, f_{2} \in \mathbb{R} .
$$

Moreover, $s_{D D T}$ is symmetric and has the following properties:

i) $s_{D D T}(f, f)=$ const for all $f \in \mathbb{R}$,

ii) $0 \leq s_{D D T}\left(f_{1}, f_{2}\right) \leq s_{D D T}(f, f)=p_{V_{1}-V_{2}}(0)$ for all $f_{1}, f_{2}, f \in \mathbb{R}$. 
Proof: By Proposition A.5 i) and Corollary A.3 i) it holds that

$$
s_{D D T}\left(f_{1}, f_{2}\right)=\int_{-\infty}^{\infty} p_{V_{1}}\left(f_{1}-u\right) p_{V_{2}}\left(f_{2}-u\right) d u=p_{V_{1}-V_{2}}\left(f_{1}-f_{2}\right) .
$$

Now, applying again Proposition A.5 i) shows that

$$
p_{V_{1}-V_{2}}\left(f_{1}-f_{2}\right)=p_{F_{1}-F_{2} \mid U_{1}-U_{2}}\left(f_{1}-f_{2} \mid 0\right) .
$$

The listed properties follow directly by Lemma A.4.

The last property guarantees that $s_{D D T}\left(f_{1}, f_{2}\right)$ is maximal whenever $f_{1}=f_{2}$ and $s_{D D T}$ is bounded so that it can be scaled to the interval $[0,1]$, i.e. the range of $s_{N L}$. For the special case that $V_{i}, i=1,2$, are normally distributed with standard deviation $\sigma$, it follows that

$$
s_{D D T}\left(f_{1}, f_{2}\right)=\frac{1}{2 \sqrt{\pi} \sigma} \exp \left(-\frac{\left|f_{1}-f_{2}\right|^{2}}{4 \sigma^{2}}\right)=\frac{1}{2 \sqrt{\pi} \sigma}\left(s_{N L}\left(f_{1}, f_{2}\right)\right)^{\frac{1}{4 \sigma^{2}}} .
$$

Hence, normalizing $s_{D D T}\left(f_{1}, f_{2}\right)$ by its maximum $c:=\max _{x, y \in \mathbb{R}} s_{D D T}(x, y)=\frac{1}{2 \sqrt{\pi} \sigma}$ leads to the weight definition

$$
w(i, j)=\prod_{k \in I}\left(\frac{s_{D D T}\left(f_{i+k}, f_{j+k}\right)}{c}\right)^{\frac{g_{a, k}}{h}}=\prod_{k \in I} s_{N L}\left(f_{i+k}, f_{j+k}\right)^{\frac{g_{a, k}}{h^{\prime}}} \quad \text { with } \quad h^{\prime}=4 \sigma^{2} h .
$$

This is just the definition of the original NL means filter with a scaled filtering parameter $h$ as similarly deduced in [7].
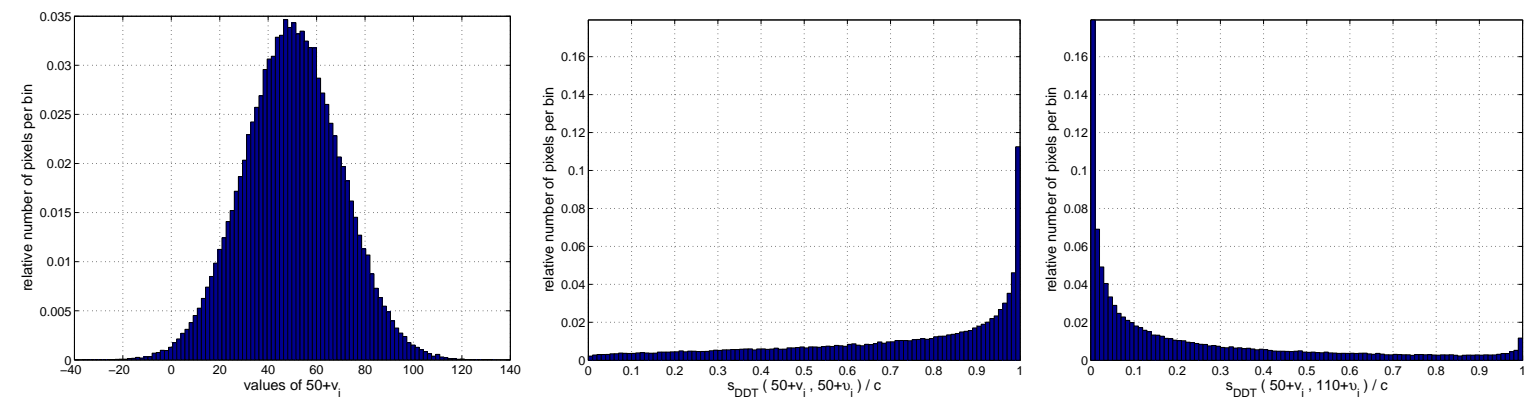

Figure 1: Left: Histogram of a constant image with gray value 50, which is corrupted by additive Gaussian noise of standard deviation 20. Middle: Histogram of $\left(s_{D D T}\left(f_{i}, \widetilde{f}_{i}\right) / c\right)_{i=1}^{N}$, where $f, \widetilde{f}$ are both constant images of gray value 50 corrupted by additive Gaussian noise of standard deviation 20. Right: Same as in the middle, but now $\widetilde{f}$ represents a constant image of gray value 110 corrupted by additive Gaussian noise of standard deviation 20.

The behavior of the similarity measure $s_{D D T}$ for additive Gaussian noise is illustrated in Figure 1. The histogram on the left shows the distribution of the gray values of a constant image of gray value 50 corrupted by additive Gaussian noise of standard deviation 20. Next, the distribution of the values $s_{D D T}\left(f_{i}, \widetilde{f}_{i}\right) / c, i=1, \ldots, N$, is depicted for the case that both images are corrupted versions of the same constant gray value image. As expected, most 
values are close to one, i.e. $s_{D D T} / c$ detected that the corresponding noisy pixels belong to the same noise free pixel. Only a few values are close to zero, which means that the measure did not recognize that also these noisy pixels had the same initial gray value. For the histogram on the right, different gray values have been used to generate the noisy images. Here, most values $s_{D D T}\left(f_{i}, \widetilde{f}_{i}\right) / c$ are close to zero and only few pixels are falsely detected to correspond to the same noise free pixel.

\subsection{Properties in the presence of multiplicative noise}

Next, we want to investigate the case of multiplicative noise. For this reason, suppose again that $V_{i}, i=1,2$, are independent identically distributed random variables, which follow some noise distribution. Now, let $f_{i}$ be corrupted by multiplicative noise, i.e. $f_{i}:=u_{i} \cdot v_{i}$ and

$$
F_{i}:=U_{i} V_{i}, \quad i=1,2,
$$

where each $v_{i}$ is again a realization of the random variable $V_{i}$. All $U_{i}, V_{i}, i=1,2$ are further considered to be pairwise independent and we suppose that $p_{U_{i}}(x)=0, p_{V_{i}}(x)=0$ for $x<0$, i.e. $F_{i}>0$ almost surely, as it is usually the case in imaging applications facing multiplicative noise.

Under these preliminaries, we obtain using Proposition A.5 ii) that for $f_{1}, f_{2}$ with $p_{F_{i}}\left(f_{i}\right)>0$, $i=1,2$, and $S=\operatorname{supp}\left(p_{U_{i}}\right) \subseteq \mathbb{R}_{\geq 0}$ the 'similarity probability' of Deledalle et al. is given by

$$
\begin{aligned}
p_{U_{1}-U_{2} \mid\left(F_{1}, F_{2}\right)}\left(0 \mid f_{1}, f_{2}\right) & =\int_{S} \frac{p_{U_{1}}(u) p_{U_{2}}(u)}{p_{F_{1}}\left(f_{1}\right) p_{F_{2}}\left(f_{2}\right)} p_{F_{1} \mid U_{1}}\left(f_{1} \mid u\right) p_{F_{2} \mid U_{2}}\left(f_{2} \mid u\right) d u \\
& =\int_{S} \frac{p_{U_{1}}(u) p_{U_{2}}(u)}{p_{F_{1}}\left(f_{1}\right) p_{F_{2}}\left(f_{2}\right)} \frac{1}{u^{2}} p_{V_{1}}\left(\frac{f_{1}}{u}\right) p_{V_{2}}\left(\frac{f_{2}}{u}\right) d u
\end{aligned}
$$

which will be investigated in the examples later on. First, we deduce the following properties of $s_{D D T}$ :

Proposition 2.2. For the described multiplicative noise model with $S=\mathbb{R}_{\geq 0}$ it holds that

$$
s_{D D T}\left(f_{1}, f_{2}\right)=\int_{0}^{\infty} \frac{1}{u^{2}} p_{V_{1}}\left(\frac{f_{1}}{u}\right) p_{V_{2}}\left(\frac{f_{2}}{u}\right) d u=p_{f_{2} V_{1}-f_{1} V_{2}}(0) \quad \forall f_{1}, f_{2}>0 .
$$

In this case, $s_{D D T}$ is symmetric and has the following properties:

i) $s_{D D T}(f, f)=\frac{1}{f} p_{V_{1}-V_{2}}(0)$ for all $f=f_{1}=f_{2}>0$,

ii) $s_{D D T}$ is not bounded from above.

Proof: Equation (11) follows directly by the definition of $s_{D D T}$, Proposition A.5 ii) and Corollary A.3 i). By Corollary A.3 iv) we have for $f=f_{1}=f_{2}>0$ that

$$
s_{D D T}(f, f)=p_{f\left(V_{1}-V_{2}\right)}(0)=\frac{1}{f} p_{V_{1}-V_{2}}(0)
$$

and thus, $s_{D D T}(f, f)$ tends to infinity for $f \rightarrow 0$. 
These properties stand in sharp contrast to the additive case. The first property implies that by $s_{D D T}$, small values $f=f_{1}=f_{2}$ are always considered more likely to be generated by the same noise free pixel than bigger ones. Moreover, the unboundedness is not desirable with regard to the weight definition of a nonlocal filter, since a single pixel could get an arbitrarily large weight and dominate all others.

To see what we get for $s_{D D T}$ for concrete noise distributions and to compare its behavior to $p_{U_{1}-U_{2} \mid\left(F_{1}, F_{2}\right)}\left(0 \mid f_{1}, f_{2}\right)$, we will consider different examples. In analogy to additive Gaussian noise, it may seem nearby to start with multiplicative Gaussian noise with mean one. However, in this case the assumption $p_{V_{i}}(x)=0$ for $x<0$ is violated. Only for a very small standard deviation it can be consider at least very unlikely that a realization $v_{i}<0$ occurs as it has also been pointed out in [1]. Hence, we will not further consider this example.

\section{Example 2.3. (Multiplicative uniform noise)}

For $i=1,2$ assume that $U_{i}$ is uniformly distributed on the interval $[0, n]$ and $V_{i}$ is uniformly distributed on $[1-m, 1+m], m \in(0,1)$, i.e.

$$
p_{U_{i}}(u)=\left\{\begin{array}{ll}
\frac{1}{n} & u \in[0, n], \\
0 & \text { otherwise }
\end{array} \quad \text { and } \quad p_{V_{i}}(v)= \begin{cases}\frac{1}{2 m} & v \in[1-m, 1+m], \\
0 & \text { otherwise. }\end{cases}\right.
$$

By technical computations we obtain

$p_{U_{1}-U_{2} \mid\left(F_{1}, F_{2}\right)}\left(0 \mid f_{1}, f_{2}\right)= \begin{cases}\frac{(1+m) \min \left\{\frac{1}{f_{1}}, \frac{1}{f_{2}}\right\}-(1-m) \max \left\{\frac{1}{f_{1}}, \frac{1}{f_{2}}, \frac{1}{(1-m) n}\right\}}{\ln \left(\min \left\{\frac{(1+m) n}{f_{1}}, \frac{1+m}{1-m}\right\}\right) \ln \left(\min \left\{\frac{(1+m) n}{f_{2}}, \frac{1+m}{1-m}\right\}\right)} & \text { if } f_{1}, f_{2} \in[0,(1+m) n] \\ 0 & \text { and } \frac{f_{1}}{f_{2}} \in\left[\frac{1-m}{1+m}, \frac{1+m}{1-m}\right], \\ 0 & \text { otherwise. }\end{cases}$

In contrast, if we assume that the distribution of $U_{i}, i=1,2$, is not known and we set $S=\mathbb{R}_{\geq 0}$, it follows that

$$
s_{D D T}\left(f_{1}, f_{2}\right)= \begin{cases}\frac{1}{4 m^{2}}\left((1+m) \min \left\{\frac{1}{f_{1}}, \frac{1}{f_{2}}\right\}-(1-m) \max \left\{\frac{1}{f_{1}}, \frac{1}{f_{2}}\right\}\right) & \text { if } \frac{f_{1}}{f_{2}} \in\left[\frac{1-m}{1+m}, \frac{1+m}{1-m}\right], \\ 0 & \text { otherwise. }\end{cases}
$$

These functions have both the property that for fixed $f_{1}$ they are maximal if $f_{2}=f_{1}$. Moreover, they tend to infinity for $f_{1}=f_{2} \rightarrow 0$, i.e. they are both unbounded.

To analyze the performance of these measures with regard to our specific application we included Figure 2. On the left, the diagrams show the histogram for each measure applied to two constant images of the same gray value corrupted by multiplicative uniform noise with $m=0.4$. On the right, the same has been repeated with two constant images of significant different gray value. As we can see here, except for a scaling factor the results of the two measures are quite similar. Moreover, the histograms for the images with different initial gray values have again a significant peak at zero meaning that most pixels have been detected to belong to different noise free pixels. In contrast to Figure 1 (middle), the peaks of the histograms on the left are not at the largest obtained values of the measures, but at some intermediate values. This is not desirable with respect to a weight definition of a nonlocal filter, since it indicates that the measures can not definitely determine whether the true pixels have been the same or not for a large number of pixels. However, also for different noise distributions the observations are similar: 

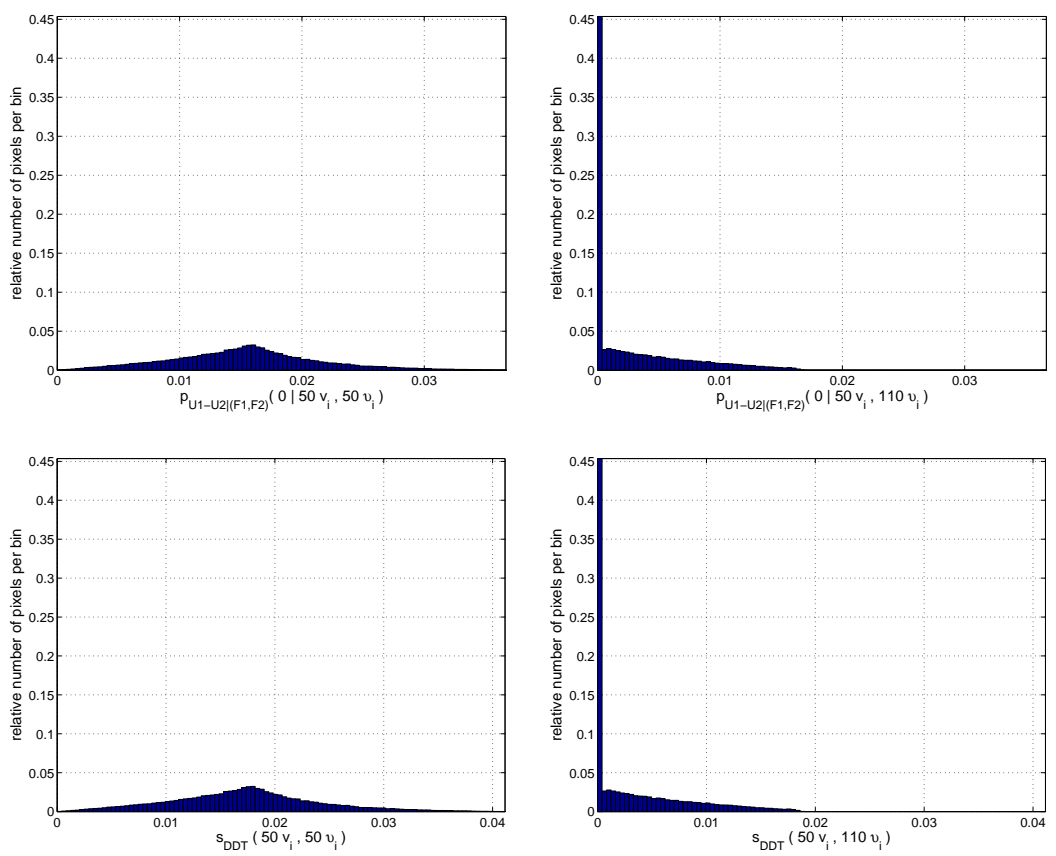

Figure 2: Left: Histograms of $\left(p_{U_{1}-U_{2} \mid\left(F_{1}, F_{2}\right)}\left(0 \mid f_{i}, \widetilde{f}_{i}\right)\right)_{i=1}^{N}$ (top) and $\left(s_{D D T}\left(f_{i}, \widetilde{f}_{i}\right)\right)_{i=1}^{N}$ (bottom), where $f, \tilde{f}$ are both constant images of gray value 50 corrupted by multiplicative uniform noise with $m=0.4$. Right: Same as on the left hand side, but now $\tilde{f}$ represents a constant image of gray value 110 corrupted by equally distributed noise.

\section{Example 2.4. (Multiplicative Gamma noise)}

For this example, let us assume that the distribution of $U_{i}$ is unknown and the noise components $V_{i}$ are Gamma distributed with

$$
p_{V_{i}}(v)=\frac{L^{L}}{\Gamma(L)} v^{L-1} \exp (-L v) 1_{\mathbb{R}_{\geq 0}}(v), \quad L \geq 1, i=1,2,
$$

where $\Gamma$ stands for the Gamma function and $1_{A}$ denotes the characteristic function, i.e. $1_{A}(v)=1$ if $v \in A$ and $1_{A}(v)=0$ otherwise. For this noise distribution we obtain for $f_{1}, f_{2}>0$ and $S=\mathbb{R}_{\geq 0}$ that

$$
s_{D D T}\left(f_{1}, f_{2}\right)=\frac{L^{2 L}}{\Gamma(L)^{2}}\left(f_{1} f_{2}\right)^{L-1} \int_{0}^{\infty} \frac{1}{u^{2 L}} \exp \left(-L \frac{f_{1}+f_{2}}{u}\right) d u .
$$

By the definition of the Gamma function, see also [7], it holds that

$$
\int_{0}^{\infty} \frac{c_{1}}{t^{b}} \exp \left(-\frac{c_{2}}{t}\right) d t=\frac{c_{1}}{c_{2}^{b-1}} \Gamma(b-1) \quad \forall c_{1} \in \mathbb{R}, c_{2}>0, b>1 .
$$

Hence, we finally obtain

$$
s_{D D T}\left(f_{1}, f_{2}\right)=L \frac{\Gamma(2 L-1)}{\Gamma(L)^{2}} \frac{\left(f_{1} f_{2}\right)^{L-1}}{\left(f_{1}+f_{2}\right)^{2 L-1}}=L \frac{\Gamma(2 L-1)}{\Gamma(L)^{2}} \frac{1}{f_{1}+f_{2}} \frac{1}{\left(2+\frac{f_{1}}{f_{2}}+\frac{f_{2}}{f_{1}}\right)^{L-1}} .
$$


One may again expect that for fixed $f_{1}$ this similarity measure is maximal if $f_{2}=f_{1}$. However, for $L>1$ and a given value $f_{1}$ it is maximal for $f_{2}=\frac{L-1}{L} f_{1}$. This is again in sharp contrast to the properties of $s_{D D T}$ in the additive case. For the special case $L=1$ we have $s_{D D T}\left(f_{1}, f_{2}\right)=\frac{1}{f_{1}+f_{2}}$. This implies that for fixed $f_{1}$ the measure $s_{D D T}\left(f_{1}, f_{2}\right)$ is large whenever $f_{2}$ is small.

Figure 3 investigates the suitability of this measure for the weight definition of a nonlocal filter. In general, the performance is very similar to the histograms in Figure 2.
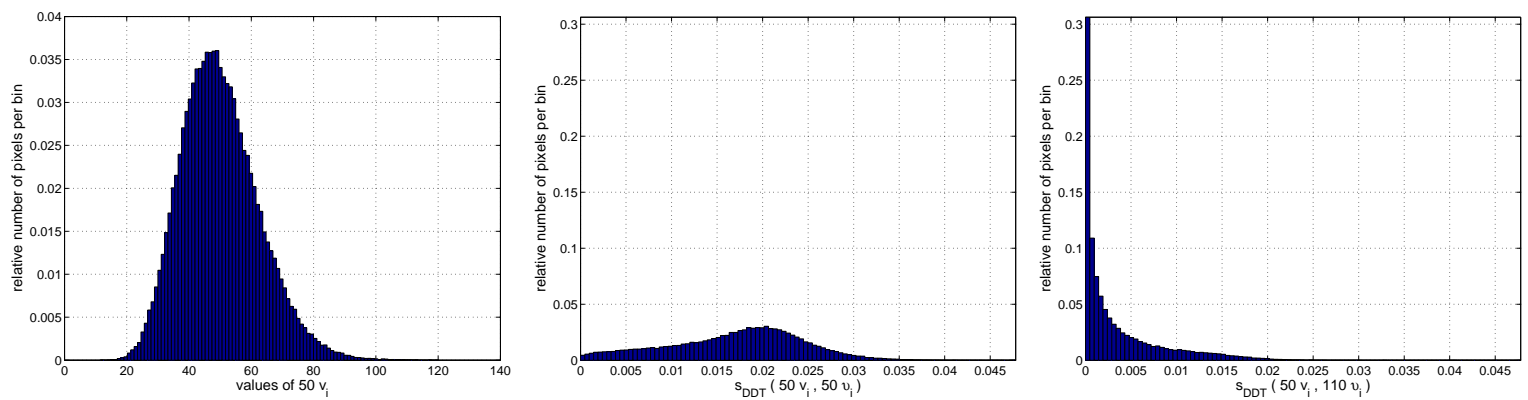

Figure 3: Left: Histogram of a constant image with gray value 50 corrupted by multiplicative Gamma noise with $L=16$. Middle: Histogram of $\left(s_{D D T}\left(f_{i}, \widetilde{f}_{i}\right)\right)_{i=1}^{N}$, where $f, \widetilde{f}$ are both constant images of gray value 50 corrupted by multiplicative Gamma noise with $L=16$. Right: Same as in the middle, but now $\widetilde{f}$ represents a constant image of gray value 110 corrupted by noise.

\section{Example 2.5. (Multiplicative Rayleigh noise)}

Finally, let for $i=1,2$ the distribution of $U_{i}$ be again unknown and suppose now that the noise components $v_{i}$ are realizations of Rayleigh distributed random variables $V_{i}$ with

$$
p_{V_{i}}(v)=\frac{v}{\theta^{2}} \exp \left(-\frac{v^{2}}{2 \theta^{2}}\right) 1_{\mathbb{R}_{\geq 0}}(v), \quad \theta>0 .
$$

Here, using again (13) we obtain for $S=\mathbb{R}_{\geq 0}$ and $f_{1}, f_{2}>0$ that

$$
s_{D D T}\left(f_{1}, f_{2}\right)=\frac{\sqrt{2}}{\theta} \Gamma\left(\frac{3}{2}\right) \frac{f_{1} f_{2}}{\left(f_{1}^{2}+f_{2}^{2}\right)^{\frac{3}{2}}}=\sqrt{\frac{\pi}{2}} \frac{1}{\theta} \frac{f_{1} f_{2}}{\left(f_{1}^{2}+f_{2}^{2}\right)^{\frac{3}{2}}} .
$$

For fixed $f_{1}$ we have in this case that $s_{D D T}\left(f_{1}, f_{2}\right)$ is maximal for $f_{2}=\frac{1}{\sqrt{2}} f_{1}$, which is again in contrast to the additive case.

Altogether, $s_{D D T}\left(f_{1}, f_{2}\right)=p_{f_{2} V_{1}-f_{1} V_{2}}(0)$ does not seem to be optimal for multiplicative noise.

\section{A new similarity measure for multiplicative noise}

To deduce a different measure for the multiplicative noise model introduced in Subsection 2.2 , let us consider the logarithmically transformed random variables $\widetilde{F}_{i}=\ln \left(F_{i}\right)$, where

$$
\underbrace{\ln \left(F_{i}\right)}_{\widetilde{F}_{i}}=\ln \left(U_{i} V_{i}\right)=\underbrace{\ln \left(U_{i}\right)}_{\widetilde{U}_{i}}+\underbrace{\ln \left(V_{i}\right)}_{\widetilde{V}_{i}}, \quad i=1,2 .
$$


The new random variables $\widetilde{F}_{i}$ follow an additive noise model now and the supports of $p_{\widetilde{U}_{i}}$, $p_{\widetilde{V}_{i}}$ may be the whole of $\mathbb{R}$. By applying (5) to these random variables the following lemma shows that this leads to $p_{\frac{U_{1}}{U_{2}} \mid\left(F_{1}, F_{2}\right)}\left(1 \mid f_{1}, f_{2}\right)$ and not $p_{U_{1}-U_{2} \mid\left(F_{1}, F_{2}\right)}\left(0 \mid f_{1}, f_{2}\right)$ :

Lemma 3.1. For $f_{1}, f_{2}>0$ with $p_{F_{i}}\left(f_{i}\right)>0$ and $\widetilde{S}=\operatorname{supp}\left(p_{\widetilde{U}_{i}}\right)$ it holds that

$$
\begin{aligned}
p_{\widetilde{U}_{1}-\widetilde{U}_{2} \mid\left(\widetilde{F}_{1}, \widetilde{F}_{2}\right)}\left(0 \mid \ln \left(f_{1}\right), \ln \left(f_{2}\right)\right) & =\int_{\widetilde{S}} \frac{p_{\widetilde{U}_{1}}(t) p_{\widetilde{U}_{2}}(t) p_{\widetilde{F}_{1} \mid \widetilde{U}_{1}}\left(\ln \left(f_{1}\right) \mid t\right) p_{\widetilde{F}_{2} \mid \widetilde{U}_{2}}\left(\ln \left(f_{2}\right) \mid t\right)}{p_{\widetilde{F}_{1}}\left(\ln \left(f_{1}\right)\right) p_{\widetilde{F}_{2}}\left(\ln \left(f_{2}\right)\right)} d t \\
& =p_{\frac{U_{1}}{U_{2}} \mid\left(F_{1}, F_{2}\right)}\left(1 \mid f_{1}, f_{2}\right) .
\end{aligned}
$$

Proof: Equation (15) follows directly by (6). Besides, we have by Proposition A.5 i) and Corollary A.2 that

$$
\begin{aligned}
\int_{\widetilde{S}} \frac{p_{\widetilde{U}_{1}}(t) p_{\widetilde{U}_{2}}(t) p_{\widetilde{F}_{1} \mid \widetilde{U}_{1}}\left(\ln \left(f_{1}\right) \mid t\right) p_{\widetilde{F}_{2} \mid \widetilde{U}_{2}}\left(\ln \left(f_{2}\right) \mid t\right)}{p_{\widetilde{F}_{1}}\left(\ln \left(f_{1}\right)\right) p_{\widetilde{F}_{2}}\left(\ln \left(f_{2}\right)\right)} d t \\
=\int_{\widetilde{S}} \frac{p_{\widetilde{U}_{1}}(t) p_{\widetilde{U}_{2}}(t) p_{\widetilde{V}_{1}}\left(\ln \left(f_{1}\right)-t\right) p_{\widetilde{V}_{2}}\left(\ln \left(f_{2}\right)-t\right)}{p_{\widetilde{F}_{1}}\left(\ln \left(f_{1}\right)\right) p_{\widetilde{F}_{2}}\left(\ln \left(f_{2}\right)\right)} d t \\
=\int_{\widetilde{S}} \frac{e^{t} p_{U_{1}}\left(e^{t}\right) e^{t} p_{U_{2}}\left(e^{t}\right) f_{1} e^{-t} p_{V_{1}}\left(f_{1} e^{-t}\right) f_{2} e^{-t} p_{V_{2}}\left(f_{2} e^{-t}\right)}{f_{1} p_{F_{1}}\left(f_{1}\right) f_{2} p_{F_{2}}\left(f_{2}\right)} d t \\
=\frac{\int_{S} \frac{1}{u} p_{U_{1}}(u) p_{U_{2}}(u) p_{V_{1}}\left(\frac{f_{1}}{u}\right) p_{V_{2}}\left(\frac{f_{2}}{u}\right) d u}{p_{F_{1}}\left(f_{1}\right) p_{F_{2}}\left(f_{2}\right)}
\end{aligned}
$$

with $S=\operatorname{supp}\left(p_{U_{i}}\right)$. Next, we set $X=\left(U_{1}, U_{2}, V_{1}, V_{2}\right), Y=\left(\frac{U_{1}}{U_{2}}, U_{2}, F_{1}, F_{2}\right), T_{1}=T_{2}=\mathbb{R}_{>0}^{4}$ and define $g: T_{1} \rightarrow T_{2}$ by $g\left(u_{1}, u_{2}, v_{1}, v_{2}\right)=\left(\frac{u_{1}}{u_{2}}, u_{2}, u_{1} v_{1}, u_{2} v_{2}\right)$. Then, Theorem A.1 yields

$$
p_{\frac{U_{1}}{U_{2}}, U_{2}, F_{1}, F_{2}}\left(x, u, f_{1}, f_{2}\right)= \begin{cases}\frac{1}{x u} p_{U_{1}, U_{2}, V_{1}, V_{2}}\left(x u, u, \frac{f_{1}}{x u}, \frac{f_{2}}{u}\right) & \text { if }\left(x, u, f_{1}, f_{2}\right)^{\mathrm{T}} \in T_{2}, \\ 0 & \text { otherwise. }\end{cases}
$$

Hence, by the pairwise independence of $U_{1}, U_{2}, V_{1}, V_{2}$ it follows that

$$
\begin{aligned}
p_{\frac{U_{1}}{U_{2}}, F_{1}, F_{2}}\left(1, f_{1}, f_{2}\right) & =\int_{-\infty}^{\infty} p_{\frac{U_{1}}{U_{2}}, U_{2}, F_{1}, F_{2}}\left(1, u, f_{1}, f_{2}\right) d u \\
& =\int_{S} \frac{1}{u} p_{U_{1}}(u) p_{U_{2}}(u) p_{V_{1}}\left(\frac{f_{1}}{u}\right) p_{V_{2}}\left(\frac{f_{2}}{u}\right) d u
\end{aligned}
$$

Inserting this in (17) leads by the definition of the conditional density to the assumption.

Now, similarly to Section 2 we omit the terms $p_{\widetilde{U}_{i}}, p_{\widetilde{F}_{i}}, i=1,2$, in (15) and suppose that $\widetilde{S}=\mathbb{R}$, which is equivalent to $S=\mathbb{R}_{\geq 0}$. With (8) this leads to

$$
\begin{aligned}
\int_{\widetilde{S}} p_{\widetilde{F}_{1} \mid \widetilde{U}_{1}}\left(\ln \left(f_{1}\right) \mid t\right) p_{\widetilde{F}_{2} \mid \widetilde{U}_{2}}\left(\ln \left(f_{2}\right) \mid t\right) d t & =p_{\widetilde{V}_{1}-\widetilde{V}_{2}}\left(\ln \left(f_{1}\right)-\ln \left(f_{2}\right)\right) \\
& =p_{\widetilde{F}_{1}-\widetilde{F}_{2} \mid \widetilde{U}_{1}-\widetilde{U}_{2}}\left(\ln \left(f_{1}\right)-\ln \left(f_{2}\right) \mid 0\right) .
\end{aligned}
$$


Setting

$$
s\left(f_{1}, f_{2}\right):=p_{\widetilde{V}_{1}-\widetilde{V}_{2}}\left(\ln \left(f_{1}\right)-\ln \left(f_{2}\right)\right),
$$

we obtain a new similarity measure, which has the following favorable properties:

Proposition 3.2. For our multiplicative noise model it holds that

$$
\begin{aligned}
& s\left(f_{1}, f_{2}\right)=p_{\frac{f_{2}}{f_{1}} \frac{V_{1}}{V_{2}}}(1)=\frac{f_{1}}{f_{2}} p_{\frac{F_{1}}{F_{2}} \mid \frac{U_{1}}{U_{2}}}\left(\frac{f_{1}}{f_{2}} \mid 1\right) \\
& =\int_{0}^{\infty} \frac{f_{1} f_{2}}{u^{3}} p_{V_{1}}\left(\frac{f_{1}}{u}\right) p_{V_{2}}\left(\frac{f_{2}}{u}\right) d u \\
& =\int_{0}^{\infty} \frac{f_{1}}{f_{2}} t p_{V_{1}}\left(\frac{f_{1}}{f_{2}} t\right) p_{V_{2}}(t) d t \quad \forall f_{1}, f_{2}>0 .
\end{aligned}
$$

Moreover, $s(\cdot, \cdot)$ is symmetric and has the following properties:
i) $s(f, f)=$ const for all $f>0$,
ii) $0 \leq s\left(f_{1}, f_{2}\right) \leq s(f, f)=p_{\frac{V_{1}}{V_{2}}}(1)$ for all $f_{1}, f_{2}, f>0$.

Proof: Corollaries A.3 i), A.2 and different variable transformations yield for $f_{1}, f_{2}>0$ that

$$
\begin{aligned}
s\left(f_{1}, f_{2}\right)=\int_{-\infty}^{\infty} p_{\widetilde{V}_{1}}\left(\ln \left(f_{1}\right)-t\right) p_{\widetilde{V}_{2}}\left(\ln \left(f_{2}\right)-t\right) d t & =\int_{0}^{\infty} \frac{f_{1} f_{2}}{u^{3}} p_{V_{1}}\left(\frac{f_{1}}{u}\right) p_{V_{2}}\left(\frac{f_{2}}{u}\right) d u \\
& =\int_{0}^{\infty} \frac{f_{1}}{f_{2}} t p_{V_{1}}\left(\frac{f_{1}}{f_{2}} t\right) p_{V_{2}}(t) d t .
\end{aligned}
$$

Moreover, it follows by Corollary A.3 iii), iv) and Proposition A.5 ii) that

$$
s\left(f_{1}, f_{2}\right)=p_{\frac{f_{2}}{f_{1}} \frac{V_{1}}{V_{2}}}(1)=\frac{f_{1}}{f_{2}} p_{\frac{V_{1}}{V_{2}}}\left(\frac{f_{1}}{f_{2}}\right)=\frac{f_{1}}{f_{2}} p_{\frac{F_{1}}{F_{2}}} \mid \frac{U_{1}}{U_{2}}\left(\frac{f_{1}}{f_{2}} \mid 1\right) .
$$

The properties of $s(\cdot, \cdot)$ follow by (18), Lemma A.4 and $s\left(f_{1}, f_{2}\right)=p_{\frac{f_{2}}{f_{1}} \frac{V_{1}}{V_{2}}}(1)$.

Note that the properties of $s(\cdot, \cdot)$ are very similar to $s_{D D T}$ in the additive case with $S=\mathbb{R}$, although (19) differs from (11) only by the factor $\frac{f_{1} f_{2}}{u}$ within the integral. Regarding (16) and $s_{D D T}$ for additive noise given in (8), our new measure is not exactly $p_{\frac{F_{1}}{F_{2}}} \mid \frac{U_{1}}{U_{2}}\left(\frac{f_{1}}{f_{2}} \mid 1\right)$, but a scaled version of it. The following examples show the advantageous properties of $s(\cdot, \cdot)$ :

Example 3.3. (Example 2.3 continued)

For $f_{1}, f_{2}>0$ and uniformly distributed random variables $U_{i}, V_{i}, i=1,2$, as in Example 2.3, we obtain by technical computations that

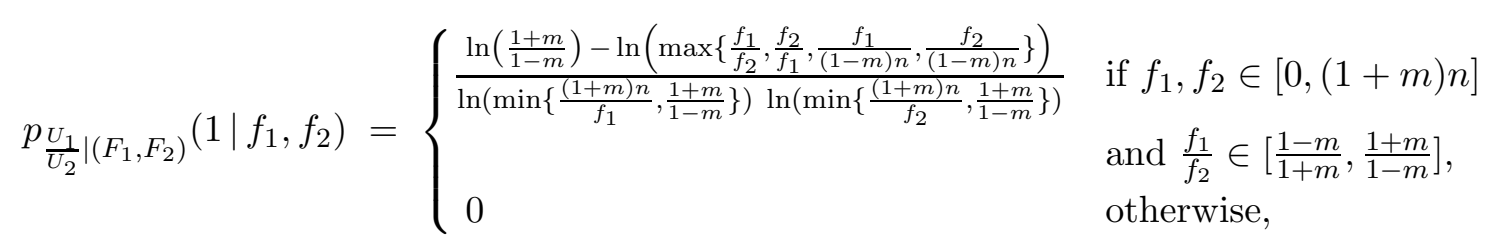


which is still not bounded, since it tends to infinity for $f_{1}=f_{2} \rightarrow(1+m) n$. Nevertheless,

$$
s\left(f_{1}, f_{2}\right)= \begin{cases}\frac{1}{8 m^{2}}\left((1+m)^{2} \min \left\{\frac{f_{1}}{f_{2}}, \frac{f_{2}}{f_{1}}\right\}-(1-m)^{2} \max \left\{\frac{f_{1}}{f_{2}}, \frac{f_{2}}{f_{1}}\right\}\right) & \text { if } \frac{f_{1}}{f_{2}} \in\left[\frac{1-m}{1+m}, \frac{1+m}{1-m}\right], \\ 0 & \text { otherwise, }\end{cases}
$$

is bounded and has a maximum of $c=\frac{1}{2 m}$.
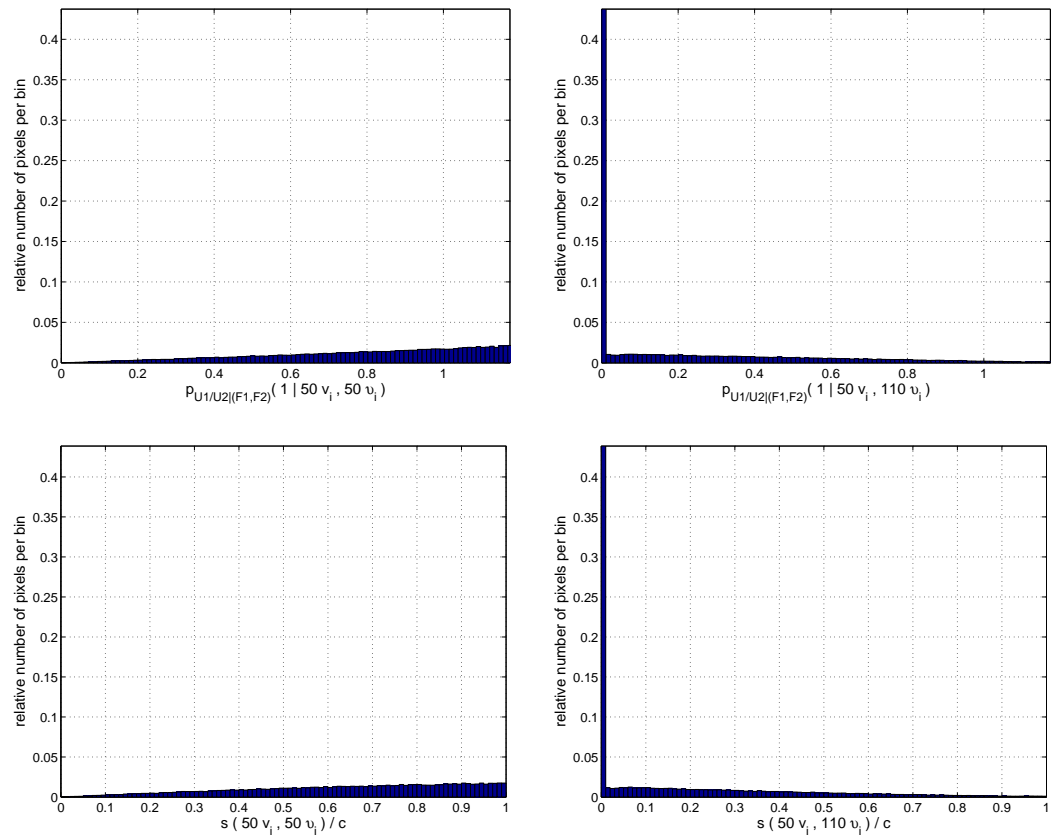

Figure 4: Left: Histograms of $\left(p_{\frac{U_{1}}{U_{2}} \mid\left(F_{1}, F_{2}\right)}\left(1 \mid f_{i}, \widetilde{f}_{i}\right)\right)_{i=1}^{N}$ (top) and $\left.\left(s\left(f_{i}, \widetilde{f}_{i}\right) / c\right)\right)_{i=1}^{N}$ (bottom), where $f, \tilde{f}$ are both constant images of gray value 50 corrupted by multiplicative uniform noise with $m=0.4$. Right: Same as on the left hand side, but now $\tilde{f}$ represents a constant image of gray value 110 corrupted by equally distributed noise.

In Figure 4 we see that except for a scaling factor the histograms for $p_{\frac{U_{1}}{U_{2}} \mid\left(F_{1}, F_{2}\right)}(1 \mid \cdot, \cdot)$ and $s(\cdot, \cdot) / c$ are quite similar for the considered images. For the two images with the same initial gray value the histograms have now their maximum at the largest obtained values. This still remains true if we consider $s(\cdot, \cdot) / c$ for multiplicative Gamma noise as our next example shows:

Example 3.4. (Example 2.4 continued)

For noise being a realization of Gamma distributed random variables $V_{i}$ it follows for $f_{1}, f_{2}>0$ using (13) that

$$
\begin{aligned}
s\left(f_{1}, f_{2}\right) & =\frac{L^{2 L}}{\Gamma(L)^{2}}\left(f_{1} f_{2}\right)^{L} \int_{0}^{\infty} \frac{1}{u^{2 L+1}} \exp \left(-L \frac{f_{1}+f_{2}}{u}\right) d u \\
& =\frac{\Gamma(2 L)}{\Gamma(L)^{2}} \frac{\left(f_{1} f_{2}\right)^{L}}{\left(f_{1}+f_{2}\right)^{2 L}}=\frac{\Gamma(2 L)}{\Gamma(L)^{2}} \frac{1}{\left(2+\frac{f_{1}}{f_{2}}+\frac{f_{2}}{f_{1}}\right)^{L}}
\end{aligned}
$$


which has a maximum of $c=\frac{\Gamma(2 L)}{\Gamma(L)^{2}} \frac{1}{4^{L}}$.

In Figure 5 we can see that for $L=16$ this new measure gives similar histograms as initially obtained in Figure 1 for additive Gaussian noise. Hence, a similar good performance can be expected if applied for nonlocal filtering.
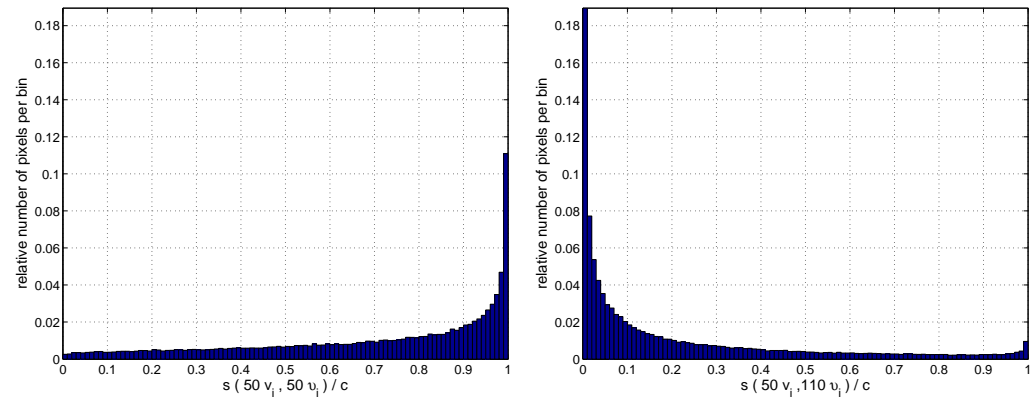

Figure 5: Left: Histogram of $\left(s\left(f_{i}, \widetilde{f}_{i}\right) / c\right)_{i=1}^{N}$, where $f, \widetilde{f}$ are both constant images of gray value 50 corrupted by multiplicative Gamma noise with $L=16$. Right: Same as on the left, but now $\tilde{f}$ represents a constant image of gray value 110 corrupted by noise.

Example 3.5. (Example 2.5 continued)

Finally, let us assume that $V_{i}, i=1,2$, follow a Rayleigh distribution. In this case, we obtain by (13) that

$$
\begin{aligned}
s\left(f_{1}, f_{2}\right) & =\frac{f_{1}^{2} f_{2}^{2}}{\theta^{4}} \int_{0}^{\infty} \frac{1}{u^{5}} \exp \left(-\frac{1}{2 \theta^{2}} \frac{f_{1}^{2}+f_{2}^{2}}{u^{2}}\right) d u \\
& =\frac{f_{1}^{2} f_{2}^{2}}{\theta^{4}} \int_{0}^{\infty} \frac{1}{2 t^{3}} \exp \left(-\frac{1}{2 \theta^{2}} \frac{f_{1}^{2}+f_{2}^{2}}{t}\right) d t \\
& =2\left(\frac{f_{1} f_{2}}{f_{1}^{2}+f_{2}^{2}}\right)^{2}=\frac{2}{\left(\frac{f_{1}}{f_{2}}+\frac{f_{2}}{f_{1}}\right)^{2}}
\end{aligned}
$$

with a maximum of $c=\frac{1}{2}$. This will be used for our numerical experiments later on.

All in all, our new similarity measure turned out to have many favorable properties, which are similar to $s_{D D T}$ facing additive noise with $S=\mathbb{R}$. To finally conclude this section we state one last observation:

Remark 3.6. If we had initially no idea how to define similarity measures for data corrupted by multiplicative noise, a first nearby approach would have been to transform $f_{1}, f_{2}>0$ logarithmically to obtain data corrupted by additive noise and to use

$$
s_{N L}\left(\ln \left(f_{1}\right), \ln \left(f_{2}\right)\right)=\exp \left(-\left(\ln \left(f_{1}\right)-\ln \left(f_{2}\right)\right)^{2}\right)=\exp \left(-\left(\ln \left(\frac{f_{1}}{f_{2}}\right)\right)^{2}\right)
$$

as a similarity measure for any $f_{1}, f_{2}$ corrupted by multiplicative noise. Interestingly, this measure can be related to the case of multiplicative Gamma noise with $L \approx 4$. In detail, 
using the Taylor approximation

$$
\ln (x)=2 \sum_{k=0}^{\infty} \frac{1}{2 k+1}\left(\frac{x-1}{x+1}\right)^{2 k+1} \approx 2 \frac{x-1}{x+1} \quad \text { for } x>0,
$$

see, e.g., [17, p. 137], we can show that

$$
\begin{aligned}
s_{N L}\left(\ln \left(f_{1}\right), \ln \left(f_{2}\right)\right) & =\exp \left(-\left(\ln \left(\frac{f_{1}}{f_{2}}\right)\right)^{2}\right)=\exp \left(\int_{1}^{f_{1} / f_{2}}-\frac{2 \ln (t)}{t} d t\right) \\
& \approx \exp \left(\int_{1}^{f_{1} / f_{2}}-\frac{4(t-1)}{t(t+1)} d t\right)=\exp \left(\left[4 \ln \left(\frac{4 t}{(t+1)^{2}}\right)\right]_{1}^{f_{1} / f_{2}}\right) \\
& =\left(\frac{4 \frac{f_{1}}{f_{2}}}{\left(\frac{f_{1}}{f_{2}}+1\right)^{2}}\right)^{4}=\left(\frac{4}{2+\frac{f_{1}}{f_{2}}+\frac{f_{2}}{f_{1}}}\right)^{4} .
\end{aligned}
$$

This last term is exactly $\frac{s\left(f_{1}, f_{2}\right)}{c}$ as given for $L=4$ in Example 3.4. Thus, $s_{N L}\left(\ln \left(f_{1}\right), \ln \left(f_{2}\right)\right)$ approximates $\frac{s\left(f_{1}, f_{2}\right)}{c}$ for data corrupted by multiplicative Gamma noise with $L \approx 4$. The quality of the approximation is shown in Figure 6. As we can see here, the graphs are very close, although there are small differences visible.
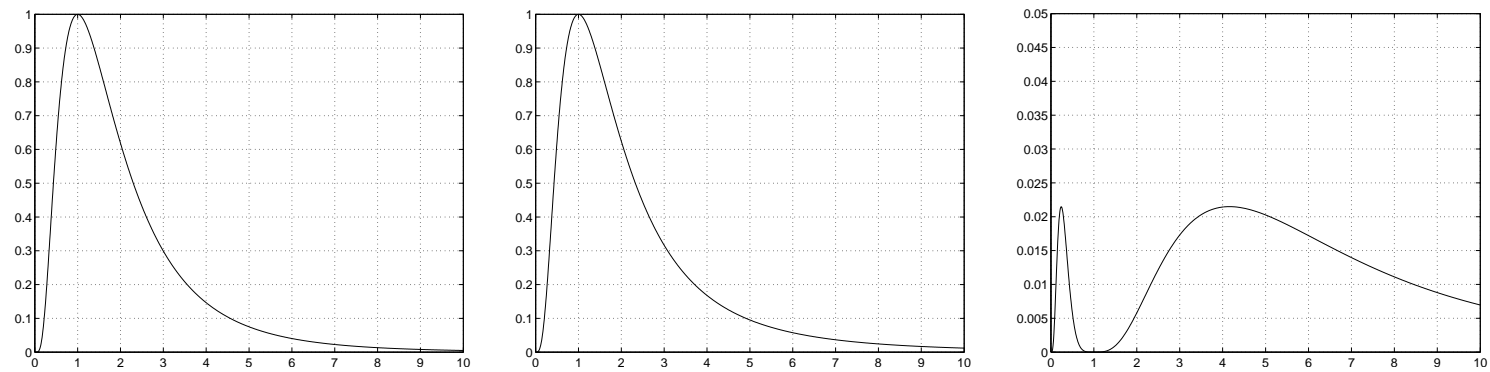

Figure 6: Graphs of the functions $t_{1}, t_{2}: \mathbb{R}_{>0} \rightarrow \mathbb{R}$ defined by $t_{1}(x)=\exp \left(-(\ln (x))^{2}\right)$ (left) and $t_{2}(x)=\left(\frac{4}{2+x+\frac{1}{x}}\right)^{4}$ (middle) as well as the graph of the difference $t_{2}-t_{1}$ (right).

\section{Nonlocal filtering in the presence of multiplicative noise}

\subsection{Nonlocal filters for multiplicative noise}

Now, we deduce our nonlocal filters by weighted maximum likelihood estimation as in [7]. Further literature on the topic can for example be found in [11, 20, 23]. Ideally, we would like to determine an estimate $\hat{u}_{i}$ of the true noise free pixels $u_{i}$ such that

$$
\hat{u}_{i}=\underset{t \in \mathbb{R}}{\operatorname{argmax}} \sum_{j \in S_{i}} \ln p_{F_{j} \mid U_{j}}\left(f_{j} \mid t\right) \quad \text { subject to } p_{U_{j}}(t)>0
$$

with $S_{i}$ being the index set of those pixels, which were generated from the same noise free pixel as $f_{i}$, i.e. $f_{j}=u_{i} v_{j}$ for all $j \in S_{i}$. Since the set $S_{i}$ is not known, we assume that for 
all $j=1, \ldots, N$ an approximation $w(i, j) \in[0,1]$ of the value of the characteristic function $1_{S_{i}}(j)$ is given and we compute

$$
\widetilde{u}_{i}:=\underset{t \in \mathbb{R}}{\operatorname{argmax}} \sum_{j=1}^{N} w(i, j) \ln p_{F_{j} \mid U_{j}}\left(f_{j} \mid t\right) \quad \text { subject to } p_{U_{j}}(t)>0 .
$$

If we set $w=w_{N L}$, it holds for additive Gaussian noise and $p_{U_{i}}>0$ that the estimates $\widetilde{u}_{i}$ are given by (1). Next, we will examine what we get for multiplicative Gamma and Rayleigh noise:

\section{Example 4.1. (Multiplicative Gamma noise)}

For our multiplicative noise model described in Subsection 2.2 and noise following a Gamma distribution we have according to (12) and Proposition A.5 ii)

$$
p_{F_{j} \mid U_{j}}\left(f_{j} \mid u_{i}\right)=\frac{L^{L}}{\Gamma(L)} \frac{f_{j}^{L-1}}{u_{i}^{L}} \exp \left(-L \frac{f_{j}}{u_{i}}\right) \quad \text { for } f_{j}, u_{i}>0 \text { with } p_{U_{j}}\left(u_{i}\right)>0 .
$$

Hence, it follows for $f_{j}>0, j=1, \ldots, N$, that

$$
\widetilde{u}_{i}=\underset{t>0, p_{U_{i}}(t)>0}{\operatorname{argmax}} \sum_{j=1}^{N} w(i, j) \ln p_{F_{j} \mid U_{j}}\left(f_{j} \mid t\right)=\underset{t>0, p_{U_{i}}(t)>0}{\operatorname{argmin}} \sum_{j=1}^{N} w(i, j)\left(\ln (t)+\frac{f_{j}}{t}\right) .
$$

In [1] Aubert and Aujol deduced similarly $H(f, u):=\sum_{i=1}^{N} \ln \left(u_{i}\right)+\frac{f_{i}}{u_{i}}$ as a data fidelity term for a variational approach to remove multiplicative Gamma noise.

If $p_{U_{i}}(t)>0$ for all $t>0$ or the distributions of the $U_{i}$ are not known, we omit the restriction $p_{U_{i}}(t)>0$ and obtain by the first order optimality condition that for $f_{j}>0, j=1, \ldots, N$, we have

$$
\widetilde{u}_{i}=\frac{1}{C_{i}} \sum_{j=1}^{N} w(i, j) f_{j} \quad \text { with } C_{i}:=\sum_{j=1}^{N} w(i, j) .
$$

Note that this is again an ordinary weighted average of the $f_{j}, j=1, \ldots, N$, like the original NL means filter in (1).

\section{Example 4.2. (Multiplicative Rayleigh noise)}

For our multiplicative noise model and Rayleigh noise it holds by (14) and Proposition A.5 ii) that

$$
p_{F_{j} \mid U_{j}}\left(f_{j} \mid u_{i}\right)=\frac{1}{\theta^{2}} \frac{f_{j}}{u_{i}^{2}} \exp \left(-\frac{1}{2 \theta^{2}}\left(\frac{f_{j}}{u_{i}}\right)^{2}\right) \quad \text { for } f_{j}, u_{i}>0 \text { with } p_{U_{j}}\left(u_{i}\right)>0
$$

Consequently, we obtain for $f_{j}>0, j=1, \ldots, N$, that

$$
\widetilde{u}_{i}=\underset{t>0, p_{U_{i}}(t)>0}{\operatorname{argmin}} \sum_{j=1}^{N} w(i, j)\left(2 \ln (t)+\frac{1}{2 \theta^{2}} \frac{f_{j}^{2}}{t^{2}}\right) .
$$

For a variational approach, $H(f, u):=\sum_{i=1}^{N} 2 \ln (t)+\frac{1}{2 \theta^{2}} \frac{f_{j}^{2}}{t^{2}}$ could also be used as a data fidelity term, where an appropriate regularization term has to be added.

If $p_{U_{i}}(t)>0$ for all $t>0$ or the distributions of the $U_{i}$ are not known, we omit again the 
restriction $p_{U_{i}}(t)>0$. For $f_{j}>0, j=1, \ldots, N$, we finally obtain by the first order optimality condition

$$
\widetilde{u}_{i}=\sqrt{\frac{1}{2 \theta^{2} C_{i}} \sum_{j=1}^{N} w(i, j) f_{j}^{2}} \quad \text { with } C_{i}:=\sum_{j=1}^{N} w(i, j) .
$$

\subsection{Adaptations of the weights}

For multiplicative noise and random variables $U_{i}$ with unknown distribution, the weights can now be defined similarly to (9) as

$$
w(i, j)=\prod_{k \in I}\left(\frac{s\left(f_{i+k}, f_{j+k}\right)}{c}\right)^{\frac{g_{a, k}}{h}},
$$

where $s\left(f_{1}, f_{2}\right)=p_{\frac{f_{2}}{f_{1}} \frac{V_{1}}{V_{2}}}(1)$ and $c=p_{\frac{V_{1}}{V_{2}}}(1)$ as defined in Section 3. As before, $g_{a}=\left(g_{a, k}\right)_{k \in I}$ represents a sampled two dimensional Gaussian kernel with mean zero and standard deviation $a$, which we normalize such that $\sum_{k \in I} g_{a, k}=1$. The parameter $h>0$ controls again the amount of filtering and the index set $I$ is set to be a squared grid of size $l \times l$ centered at 0 using reflecting boundary conditions for $f$.

Figure 7 (top) shows the histograms of the weights defined in (24) for different constant image patches of size $5 \times 5$ corrupted by multiplicative Gamma noise with $L=16$. As we can see here, multiplying the values of the similarity measure over a whole patch significantly
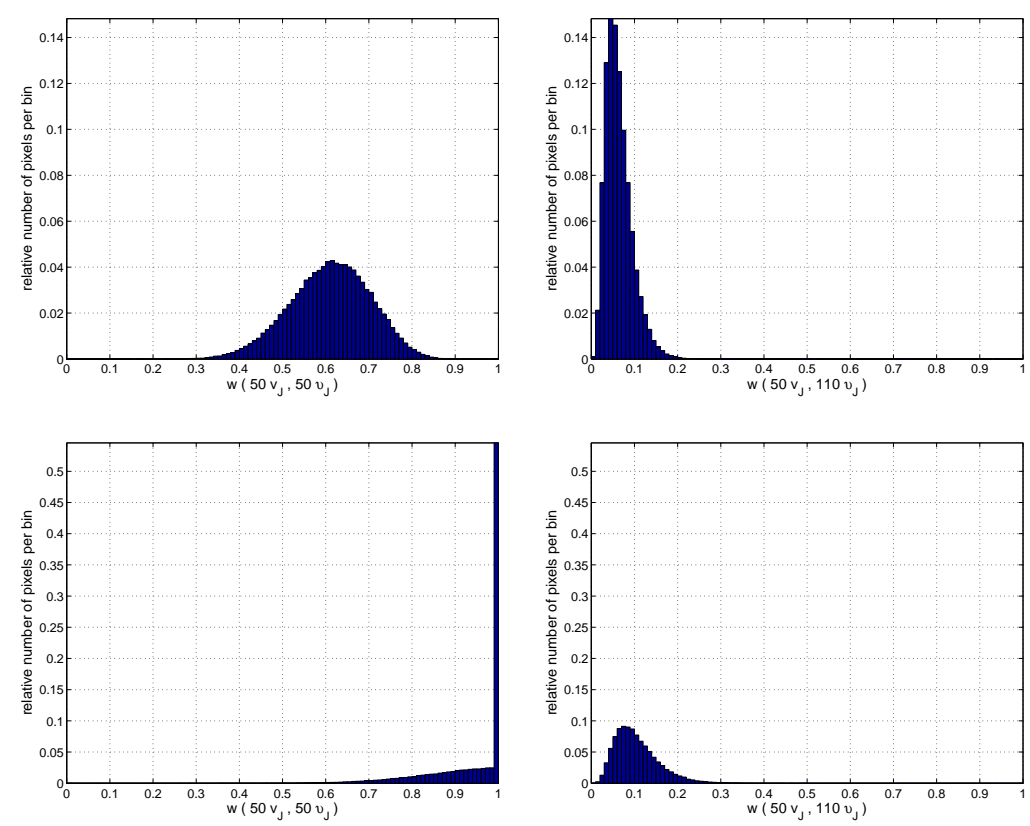

Figure 7: Histograms of the weights (24) (top) and (25) (bottom) applied to compare $N$ different image patches $f_{I}, \widetilde{f}_{I}(l=5, a=1.5, h=1, q=0)$. Left: Both $f_{I}, \widetilde{f}_{I}$ are patches of gray value 50 corrupted by multiplicative Gamma noise with $L=16$. Right: Same as on the left, but now $\widetilde{f}_{I}$ represents a constant image patch of gray value 110 corrupted by noise. 
changes the histograms compared to Figure 5. Now, the supports of the two histograms do no longer overlap, i.e. the weights for the noisy images generated from the same constant image are always larger than those computed for the constant images of significantly different gray values. Unfortunately, the histogram on the left is no longer maximal at one. Even worse, weights close to one have never been assigned.

To overcome this drawback we propose an additional adaptation of the weights inspired by the implementation of the NL means filter described at [2]. Here, we use that for random variables $X, Y$ and a continuous function $b$, where $\mathbb{E}(b(Y))$ exists, the conditional expectation of $b(Y)$ given $X=x$ is

$$
\mathbb{E}(b(Y) \mid X=x):=\int_{-\infty}^{\infty} b(y) p_{Y \mid X}(y \mid x) d y \quad \forall x \text { with } p_{X}(x)>0,
$$

see, e.g., [22, p. 168]. In detail, for two sets of random variables $F_{i+k}=U_{i+k} V_{i+k}, F_{j+k}=$ $U_{j+k} V_{j+k}, k \in I$ fulfilling the assumptions in Subsection 2.2, we set

$$
b_{k}\left(\frac{f_{i+k}}{f_{j+k}}\right):=\left(\frac{p_{\frac{f_{j+k}}{f_{i+k}} \frac{V_{i+k}}{V_{j+k}}}(1)}{c}\right)^{\frac{g_{a, k}}{h}}=\left(\frac{s\left(f_{i+k}, f_{j+k}\right)}{c}\right)^{\frac{g_{a, k}}{h}} .
$$

Assuming that the index sets $i+I, j+I$ have an empty intersection, i.e. the considered image patches are non-overlapping, we have

$$
\mu:=\mathbb{E}\left(\prod_{k \in I} b_{k}\left(\frac{F_{i+k}}{F_{j+k}}\right) \mid\left(\frac{U_{i+k}}{U_{j+k}}=1\right)_{k \in I}\right)=\prod_{k \in I} \mathbb{E}\left(b_{k}\left(\frac{F_{i+k}}{F_{j+k}}\right) \mid \frac{U_{i+k}}{U_{j+k}}=1\right) .
$$

The definition of the conditional expectation and Proposition A.5 ii) yield

$$
\mathbb{E}\left(b_{k}\left(\frac{F_{i+k}}{F_{j+k}}\right) \mid \frac{U_{i+k}}{U_{j+k}}=1\right)=\mathbb{E}\left(b_{k}\left(\frac{V_{i+k}}{V_{j+k}}\right)\right)=\int_{0}^{\infty} b_{k}(t) p_{\frac{V_{i+k}}{V_{j+k}}}(t) d t
$$

and thus, we finally obtain

$$
\mu=\prod_{k \in I} \int_{0}^{\infty} b_{k}(t) p_{\frac{V_{i+k}}{V_{j+k}}}(t) d t
$$

Since $w(i, j)$ is a realization of $\prod_{k \in I} b_{k}\left(\frac{F_{i+k}}{F_{j+k}}\right)$, the variable $\mu$ describes the value we can expect for $w(i, j)$ considering (non-overlapping) image patches which have been generated from the same noise free patch. By some technical computations we obtain for multiplicative Gamma noise

$$
\mu=\prod_{k \in I} 4^{L g_{a, k} / h} \frac{\Gamma(2 L)}{\Gamma(L)^{2}} \frac{\Gamma\left(L\left(1+\frac{g_{a, k}}{h}\right)\right)^{2}}{\Gamma\left(2 L\left(1+\frac{g_{a, k}}{h}\right)\right)}
$$

and for multiplicative Rayleigh noise

$$
\mu=\prod_{k \in I} 4^{g_{a, k} / h} \frac{\Gamma\left(1+\frac{g_{a, k}}{h}\right)^{2}}{\Gamma\left(2\left(1+\frac{g_{a, k}}{h}\right)\right)} .
$$


Next, we set

$$
w_{\mu, q}(i, j):= \begin{cases}1 & \text { if } w(i, j) \geq \mu \\ \frac{w(i, j)}{\mu} & \text { if } q \mu \leq w(i, j)<\mu \\ 0 & \text { otherwise }\end{cases}
$$

with $q \in[0,1)$ and use these weights in our nonlocal filters deduced from (20). Note that for overlapping image patches, $\mu$ is used as an approximation of the true expectation value here. The effect of this modification in contrast to the weights (24) can be seen in Figure 7 (bottom). The histogram for the image patches generated from the same noise free patch has now a significant peak at one. By setting, e.g., $q=0.5$ we could additionally achieve that all weights of the right histogram are set to zero and thus, the corresponding patches would have no effect if used in a nonlocal filter. On the contrary, the weights of the left histogram would not be effected. For our numerical examples in the subsequent section, $q$ has been set by hand. Alternatively, a statistical estimate for $q$ can for example be found by considering $1-F^{-1}(1-\beta)$ for a value $\beta \in(0,1)$ very close to one, where $F$ is the cumulative distribution function of $r_{\mu}\left(\prod_{k \in I} b_{k}\left(\frac{V_{i+k}}{V_{j+k}}\right)\right)$ with

$$
r_{\mu}(x)= \begin{cases}1 & \text { if } x \geq \mu \\ \frac{x}{\mu} & \text { otherwise }\end{cases}
$$

and $F^{-1}(\alpha)=\inf \{x \in \mathbb{R}: F(x) \geq \alpha\}$ denotes the corresponding $\alpha$-quantile. Thus, $q$ would be set to the maximal value such that the weights $w_{\mu, q}(i, j)$ do not change compared to $w_{\mu, 0}(i, j)$ for $100 \cdot \beta$ percent of the image patches $f_{i+I}, f_{j+I}$ obtained from the same noise free patch.

As usually done, we finally restrict the number of patches being compared to a so-called similarity window. Thus, we set all weights $w(i, j), w_{\mu, q}(i, j)$ automatically to zero if pixel $j$ is outside of a squared image region of size $\omega \times \omega$ centered at pixel $i$. This reduces the computational costs as well as the risk of falsely assigning nonzero weights to a large number of patches.

\subsection{Updating the similarity neighborhoods}

In [7] Deledalle et al. suggest to refine the weights of their nonlocal filters iteratively using the previous result $u^{(r-1)}$. To get the next iterate $u^{(r)}$, the filter is again applied to the initial noisy image using the new weights. In the following, we apply a variant of this updating strategy. The first major difference is that we perform only one updating step. For this second step we use within the similarity windows for $i \neq j$ the weights

$$
\widetilde{w}_{i, j}\left(u^{(1)}\right)=\exp \left(-\frac{1}{d} \sum_{k \in \widetilde{I}} g_{\widetilde{a}, k} K_{\mathrm{sym}}\left(p_{F_{i+k} \mid U_{i+k}}\left(\cdot \mid u_{i+k}^{(1)}\right), p_{F_{j+k} \mid U_{j+k}}\left(\cdot \mid u_{j+k}^{(1)}\right)\right)\right)
$$

instead of the ones defined in the former subsection and set $\widetilde{w}_{i, i}\left(u^{(1)}\right)=\max _{j} \widetilde{w}_{i, j}\left(u^{(1)}\right)$. Here, $d$ is a positive parameter and $g_{\widetilde{a}}=\left(g_{\widetilde{a}, k}\right)_{k \in \widetilde{I}}$ represents again a sampled two dimensional Gaussian kernel with mean zero, but with standard deviation $\widetilde{a}$ now. As before, $g_{\widetilde{a}}$ is normalized 
such that $\sum_{k \in \widetilde{I}} g_{\widetilde{a}, k}=1$. Moreover, the index set $\widetilde{I}=\widetilde{l} \times \widetilde{l}$ may vary from $I$. Usually, we choose $\widetilde{a}<a$ and $\tilde{l}<l$. Furthermore,

$$
K_{\mathrm{sym}}\left(p_{X}, p_{Y}\right):=\int_{-\infty}^{\infty}\left(p_{X}(t)-p_{Y}(t)\right) \ln \left(\frac{p_{X}(t)}{p_{Y}(t)}\right) d t
$$

denotes the symmetric Kullback-Leibler divergence of the density functions $p_{X}$ and $p_{Y}$. The idea for this updating scheme was originally taken from [20]. Here, Polzehl and Spokoiny used the ordinary Kullback-Leibler divergence of $p_{F_{i} \mid U_{i}}\left(\cdot \mid u_{i}^{(r-1)}\right)$ and $p_{F_{j} \mid U_{j}}\left(\cdot \mid u_{j}^{(r-1)}\right)$ to test for the hypotheses $u_{i}=u_{j}$ using estimates $u_{i}^{(r-1)}, u_{j}^{(r-1)}$ of $u_{i}, u_{j}$, respectively.

Since our numerical experiments in the next section deal with multiplicative Gamma and Rayleigh noise, we compute the symmetric Kullback-Leibler divergence for these two examples:

\section{Example 4.3. (Multiplicative Gamma noise)}

Let us assume that $p_{U_{i}}(x)>0$ for all $x>0$. Then, we obtain by straightforward calculation using (21) and (13) that the sought symmetric Kullback-Leibler divergence is given by

$$
K_{\mathrm{sym}}\left(p_{F_{i} \mid U_{i}}\left(\cdot \mid u_{i}^{(1)}\right), p_{F_{j} \mid U_{j}}\left(\cdot \mid u_{j}^{(1)}\right)\right)=L \frac{\left(u_{i}^{(1)}-u_{j}^{(1)}\right)^{2}}{u_{i}^{(1)} u_{j}^{(1)}} \quad \text { for } u_{i}^{(1)}, u_{j}^{(1)}>0 .
$$

\section{Example 4.4. (Multiplicative Rayleigh noise)}

Assume again that $p_{U_{i}}(x)>0$ for all $x>0$. In case of Rayleigh noise similar calculations yield that the symmetric Kullback-Leibler divergence is

$$
K_{\text {sym }}\left(p_{F_{i} \mid U_{i}}\left(\cdot \mid u_{i}^{(1)}\right), p_{F_{j} \mid U_{j}}\left(\cdot \mid u_{j}^{(1)}\right)\right)=\frac{\left(\left(u_{i}^{(1)}\right)^{2}-\left(u_{j}^{(1)}\right)^{2}\right)^{2}}{\left(u_{i}^{(1)}\right)^{2}\left(u_{j}^{(1)}\right)^{2}} \quad \text { for } u_{i}^{(1)}, u_{j}^{(1)}>0 .
$$

\section{$5 \quad$ Numerical results}

In the following, we present different examples demonstrating the very good performance of our nonlocal filters. These filters have been implemented in MATLAB and the parameters are chosen with respect to the best visual results. Furthermore, all images, especially the noisy ones, are displayed in the gray scale of the original image to have a consistent coloring for each example. To this purpose, all image values outside of the range of the original image are projected on this range.

Our first three examples show different reconstructions of images contaminated by multiplicative Gamma noise. The original and noisy images presented in Figure 8 are the same as those presented in [24, Fig. 5 and 6$]$ so that the results are directly comparable.

To obtain the restored image in Figure 9 (right) we used the weighted average filter derived in (22) with weights (25). As we see here, already without an additional updating of the weights we obtain a very good reconstruction, which is superior to the results by the variational Idivergence - TV (left) and I-divergence - NL means methods (middle) taken from [24].

Also in our next example the reconstructions in Figure 10 (bottom middle and right) outperform the result by the I-divergence - TV method at top left. At top middle we included a 

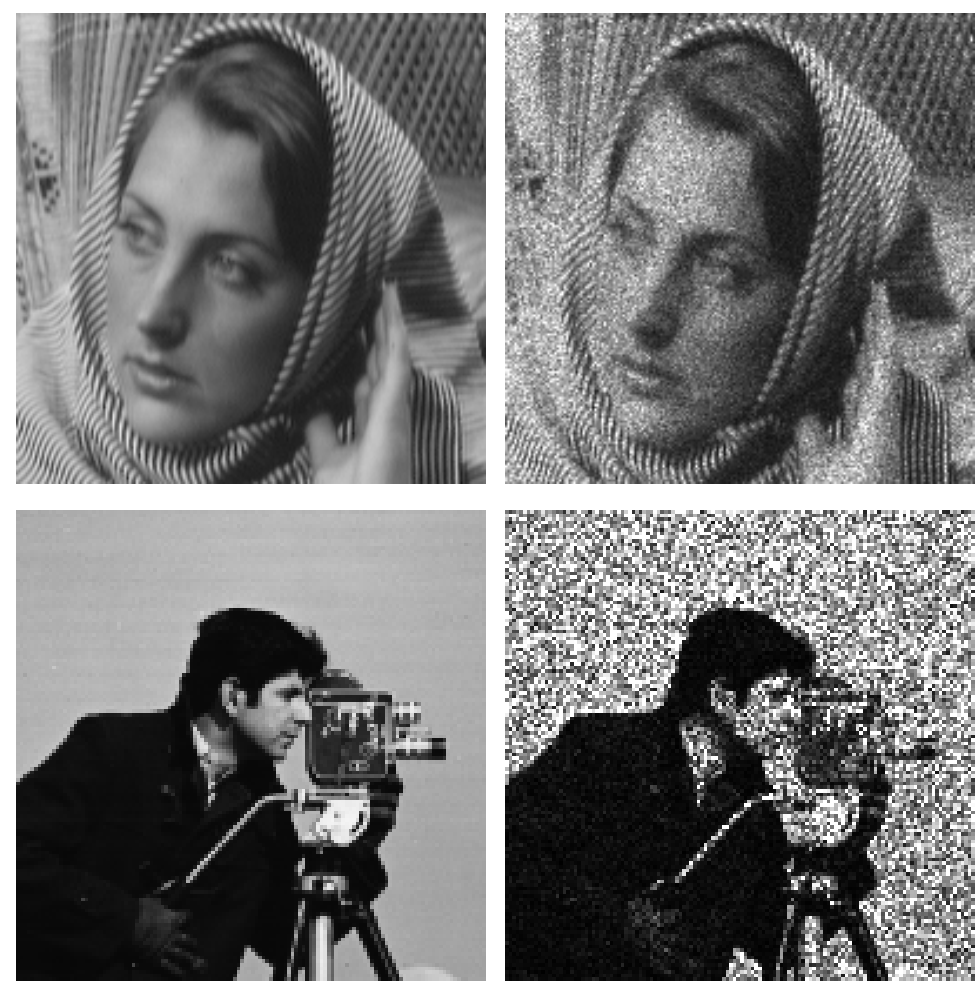

Figure 8: Left: Original images with values in $[0,255]$. Right: Noisy versions corrupted by multiplicative Gamma noise with $L=25$ (top) and $L=4$ (bottom).

reconstruction by the original NL means filter using for the patch comparison $s_{N L}(\ln (\cdot), \ln (\cdot))$ instead of $s_{N L}$. Here, the nonzero weights have again been restricted to a similarity window and the patches are chosen in the same way as for our filters. As predicted by Remark 3.6, this result is nearly the same as the one by our nonlocal filter (22) using the weights (24), which can be found at top right. Note that we have chosen slightly different values $h$ to get even more similar results. By the definition of the weights we can see that for appropriate values $h$ this can also be achieved for multiplicative Gamma noise with $L \neq 4$.

The results at Figure 10 (bottom left and middle) show the effect of applying (22) with weights $w_{\mu, 0}(i, j)$ or $w_{\mu, q}(i, j)$ instead of $w(i, j)$. By replacing $w(i, j)$ by $w_{\mu, 0}(i, j)$ we achieved an additional suppression of the noise especially in the background. Choosing further $w_{\mu, q}(i, j)$ with an appropriate value $q$ helped to improve the contrast, e.g., visible at the camera. Besides, it led to sharper edges and contours. By the final updating step applied at Figure 10 (bottom right) we further improved the contrast and small amounts of possibly remained noise are finally removed.

Next, we restored the noisy image of [10, Fig. 8] shown in Figure 11 (top right). Note that we have displayed the corrupted image in a different way. For a better comparison we include the peak signal to noise ratios (PSNR) and mean absolute-deviation errors (MAE) of the results 

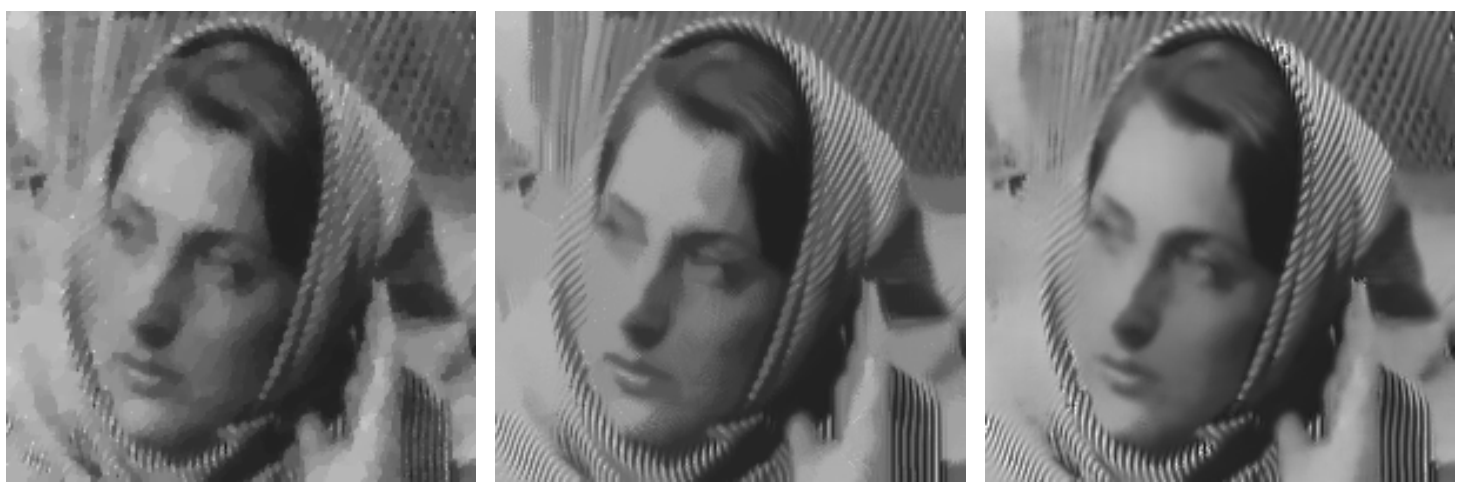

Figure 9: Results for the noisy image at Figure 8 (top right) after applying the I-divergence - TV (left) and I-divergence - NL model (middle) as shown in [24, Fig. 5] and a result by our nonlocal filter (22) using the weights (25) with $l=7, \omega=21, a=2.5, h=1, q=0.6$ (right).
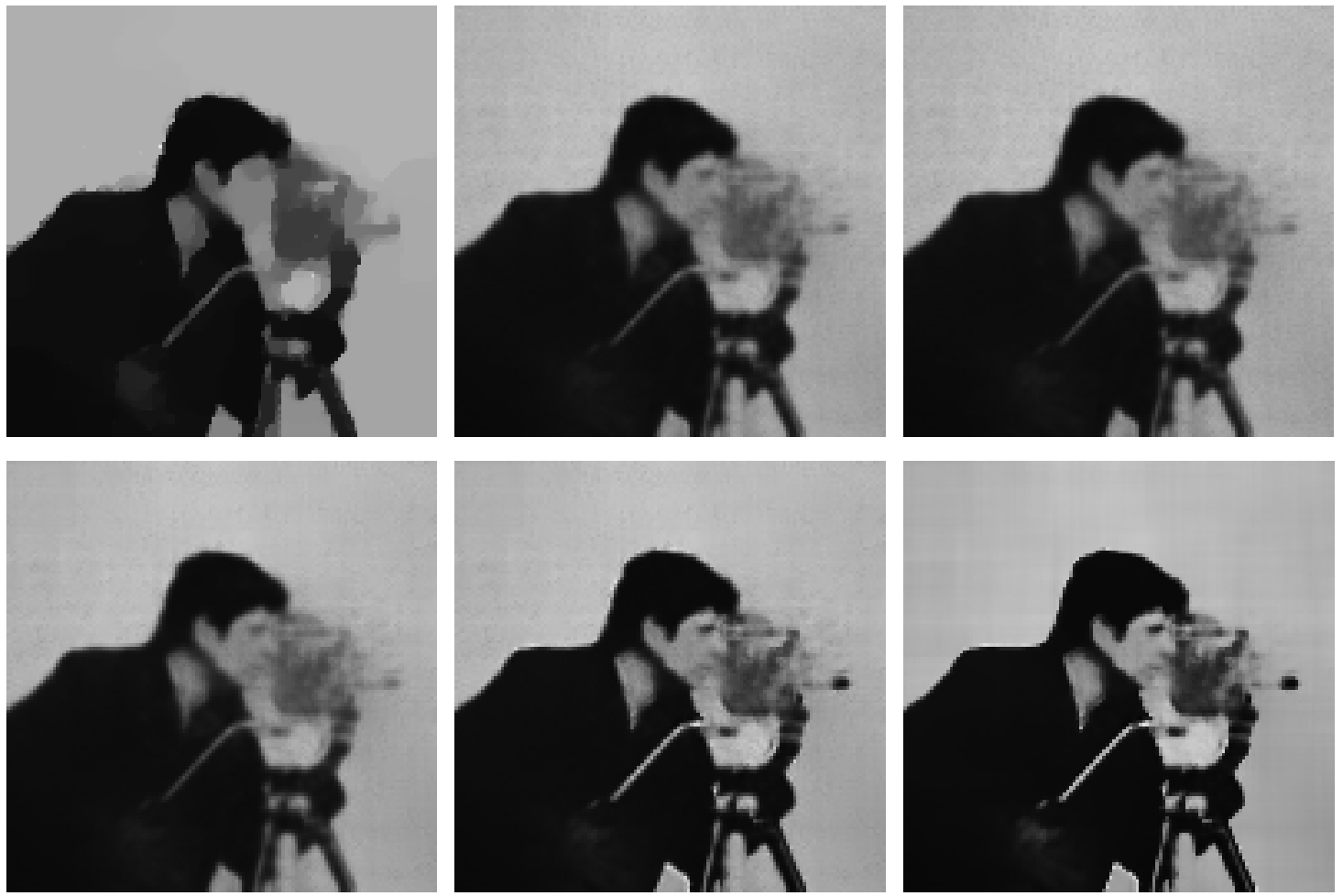

Figure 10: Results for the noisy image at bottom right of Figure 8. Top: Restored images by I-divergence - TV as presented in [24, Fig. 5] (left), by the original NL means filter using $s_{N L}(\ln (\cdot), \ln (\cdot))$ instead of $s_{N L}$ with $l=7, \omega=29, a=1.5, h=1.3$ (middle) and by our new nonlocal filter (22) using the weights (24) with $l=7, \omega=29, a=1.5, h=1$ (right). Bottom: Results by our nonlocal filter using (25) with additional parameter $q=0$ (left), $q=0.35$ (middle) and finally after an additional updating step with $\widetilde{l}=3, \widetilde{a}=0.5, d=0.25$ (right). 

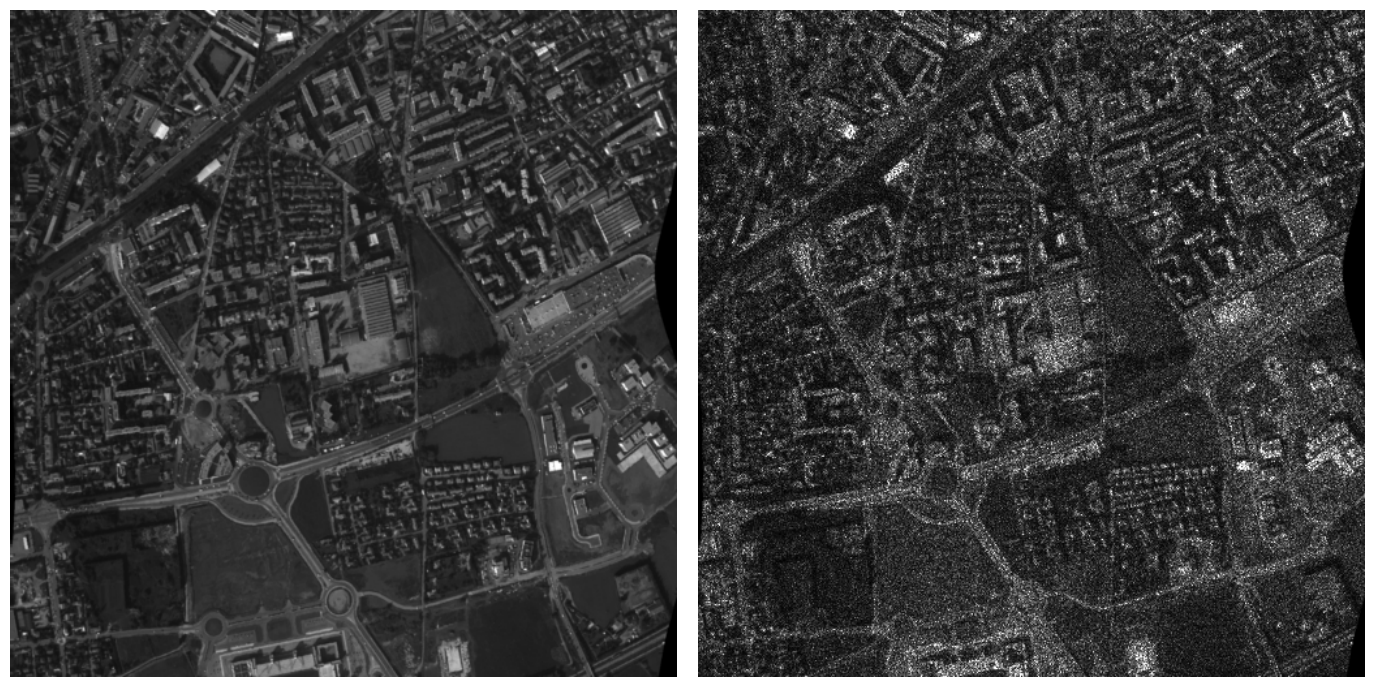

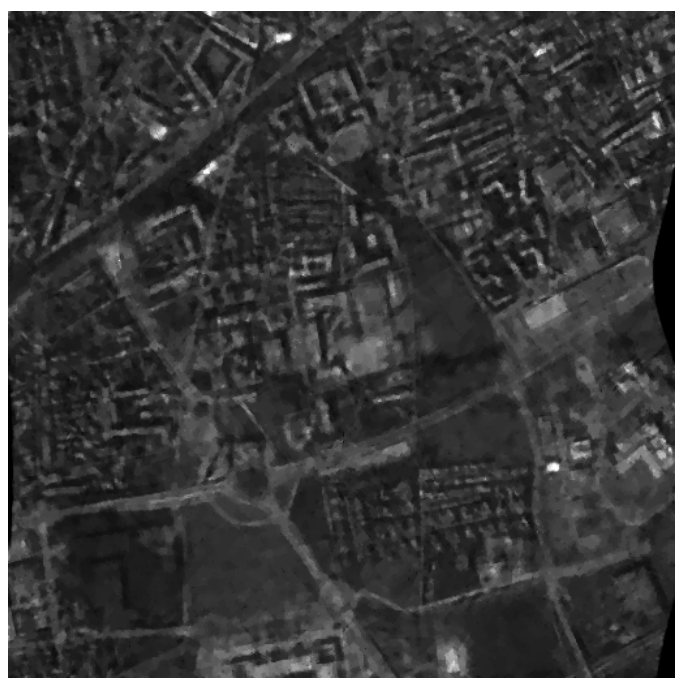

$\mathrm{PSNR}=25.84, \mathrm{MAE}=9.09$

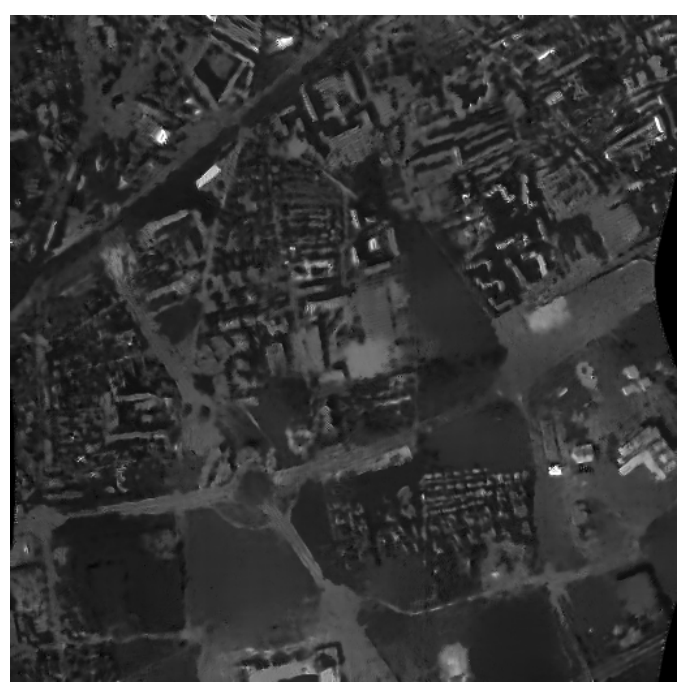

$\mathrm{PSNR}=26.01, \mathrm{MAE}=8.60$

Figure 11: Top: Original image of the French city of Nîmes $(512 \times 512)$ with values in $[1,256]$ (left), cf. [10], and noisy image corrupted by multiplicative Gamma noise with $L=4$ (right). Bottom: Result by the hybrid method of Durand et al. shown in [10, Fig. 8] (left) and the result by our nonlocal filter (22) using (25) and an additional updating step with $l=7$, $\omega=29, a=2, h=0.5, q=0.7, \widetilde{l}=5, \widetilde{a}=1, d=0.1$ (right). 
defined by

$$
\operatorname{PSNR}=10 \ln _{10} \frac{N|\max u-\min u|^{2}}{\|\widetilde{u}-u\|_{2}} \quad \text { and } \quad \operatorname{MAE}=\frac{1}{N}\|\widetilde{u}-u\|_{1} .
$$

Here, $u$ denotes the original noise free image, $\widetilde{u}$ the reconstruction and $N$ the number of pixels of each image. As a direct comparison shows, our obtained reconstruction is superior or at least competitive to the results obtained by different methods in [10, Fig. 8]. In this paper the best result was obtained by the proposed hybrid multiplicative noise removal method, which combines variational and sparsity-based shrinkage methods involving curvelets as well as TV regularization. The result of this method is presented at Figure 11 (bottom left).

To conclude this section, we present a reconstruction of an image corrupted by multiplicative Rayleigh noise in Figure 12. Here, we have applied the nonlocal filter (23) with weights (25) adapted to this type of noise.

\section{Conclusions}

In this paper we have presented a new noise adapted similarity measure for comparing data corrupted by multiplicative noise. This measure is deduced by an appropriate probability density function and its good properties have been studied theoretically as well as by numerical examples. Moreover, we have shown how this measure can be incorporated into the weight definition of nonlocal filters, which leads to very good denoising results as demonstrated for images corrupted by multiplicative Gamma and Rayleigh noise.

Nevertheless, the use of our measure is not restricted to nonlocal filtering. It could also be incorporated into appropriate variational methods or used for different applications such as inpainting and segmentation, see, e.g., [12, 13, 14, 24]. Besides, it could be considered for block matching in registration problems, cf. [19]. These are only a few other fields of application, which might be topics of future research.

\section{Acknowledgment}

The authors would like to thank the authors of [10], in particular Mila Nikolova, for providing the original and noisy images as well as the result of their method presented in Figure 11. Moreover, they thank Marc Sigelle and Florence Tupin for the initial data used in Figure 12.

\section{A Appendix}

To compute the density functions occurring in this paper we have used the following results. Here, all random variables are supposed to be real-valued and continuous.

Theorem A.1. (Jacobi's Transformation Formula, cf. [6, p. 331], [16, p. 92f])

Let $X:=\left(X_{1}, \ldots, X_{n}\right)$ have the joint probability density function $p_{X}$ and let $T_{1} \subseteq \mathbb{R}^{n}$ be an open set with $P\left(X \in T_{1}\right)=1$. For $T_{2} \subseteq \mathbb{R}^{n}$ let $g: T_{1} \rightarrow T_{2}$ be an injective function which has a continuously differentiable inverse $g^{-1}$ on $T_{2}$ with non-vanishing Jacobian. Then, the density of $Y=g(X)$ is given by

$$
p_{Y}(y)= \begin{cases}p_{X}\left(g^{-1}(y)\right)\left|\operatorname{det} J_{g^{-1}}(y)\right| & \text { if } y \in T_{2} \\ 0 & \text { otherwise }\end{cases}
$$



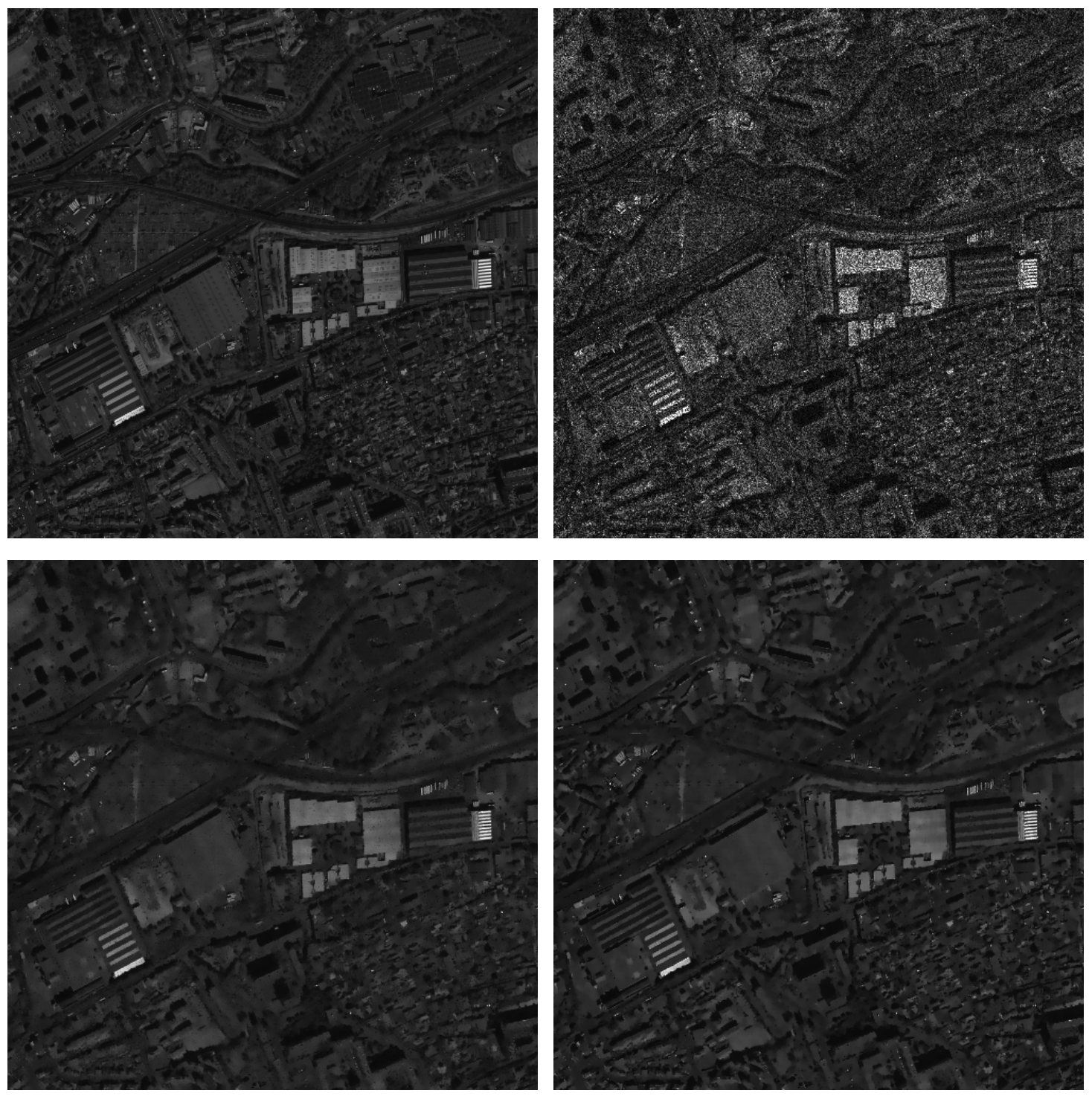

$\mathrm{PSNR}=30.41, \mathrm{MAE}=42.88$

$\mathrm{PSNR}=30.83, \mathrm{MAE}=39.06$

Figure 12: Top: Original image $(1500 \times 1500)$, copyright [21], with values in $[1,2047]$ (left) and noisy version corrupted by multiplicative Rayleigh noise with $\theta=1$ (right). Bottom: Results by our nonlocal filter (23) using (25) with $l=7, \omega=21, a=2, h=0.4, q=0.6$ (left) and after an additional updating step with $\widetilde{l}=5, \widetilde{a}=1.5, d=0.05$ (right). Note that the displayed images have been subsampled to the size $500 \times 500$ to better meet the standard screen and printer resolutions. 
This theorem immediately implies the next two corollaries.

Corollary A.2. Let $X$ be a random variable with probability density function $p_{X}$, where $p_{X}$ vanishes outside the interval $[0,+\infty)$. Then, the probability density function of $Y=\ln (X)$ is given by

$$
p_{Y}(y)=\exp (y) p_{X}(\exp (y)) .
$$

Corollary A.3. (Cf. [6, p. 336] and [15, p. 109])

For constants $c, c_{1}, c_{2} \neq 0$ and independent random variables $X, X_{1}, X_{2}$, we obtain the following probability density functions:

i)

$$
p_{c_{1} X_{1}+c_{2} X_{2}}(y)=\frac{1}{\left|c_{1} c_{2}\right|} \int_{-\infty}^{\infty} p_{X_{1}}\left(\frac{t}{c_{1}}\right) p_{X_{2}}\left(\frac{y-t}{c_{2}}\right) d t
$$

ii)

$$
p_{c X_{1} X_{2}}(y)=\int_{-\infty}^{\infty} \frac{1}{|c t|} p_{X_{1}}\left(\frac{t}{c}\right) p_{X_{2}}\left(\frac{y}{t}\right) d t
$$

iii)

$$
p_{c \frac{X_{1}}{X_{2}}}(y)=\int_{-\infty}^{\infty}\left|\frac{t}{c}\right| p_{X_{1}}\left(\frac{y t}{c}\right) p_{X_{2}}(t) d t
$$

iv)

$$
p_{c X}(y)=\frac{1}{|c|} p_{X}\left(\frac{y}{c}\right)
$$

v)

$$
p_{\frac{1}{X}}(y)=\frac{1}{y^{2}} p_{X}\left(\frac{1}{y}\right) \quad \text { for } y \neq 0 .
$$

Setting $c_{1}=-c_{2}=1$ in Corollary A.3 i) we obtain the following lemma:

Lemma A.4. For independent random variables $X_{1}, X_{2}$, the probability density function $p_{X_{1}-X_{2}}$ has the following properties:

i) $p_{X_{1}-X_{2}}(y)=p_{X_{2}-X_{1}}(-y)$,

ii) for identically distributed random variables $X_{1}, X_{2}$ we have

$$
p_{X_{1}-X_{2}}(y) \leq p_{X_{1}-X_{2}}(0) \quad \forall y \in \mathbb{R} .
$$

Proof: Assertion i) follows directly from Corollary A.3 i). To prove ii) we use in addition the Cauchy-Schwarz inequality:

$$
\begin{aligned}
p_{X_{1}-X_{2}}(y)=\int_{-\infty}^{\infty} p_{X_{1}}(t) p_{X_{2}}(t-y) d t & \leq \sqrt{\int_{-\infty}^{\infty}\left(p_{X_{1}}(t)\right)^{2} d t \int_{-\infty}^{\infty}\left(p_{X_{2}}(t-y)\right)^{2} d t} \\
& =\int_{-\infty}^{\infty}\left(p_{X_{1}}(t)\right)^{2} d t=p_{X_{1}-X_{2}}(0) .
\end{aligned}
$$


Finally, Theorem A.1 and the definition of the conditional density yield the following result for the sum, respectively the product of two independent random variables:

Proposition A.5. Let $X, Y$ be independent random variables.

i) If $Z:=X+Y$, then for any $x$ with $p_{X}(x)>0$ the conditional density function of $Z$ given $X=x$ is

$$
p_{Z \mid X}(z \mid x)=p_{Y}(z-x) .
$$

ii) If $Z:=X Y$, then for any $x \neq 0$ with $p_{X}(x)>0$ the conditional density function of $Z$ given $X=x$ is

$$
p_{Z \mid X}(z \mid x)=\frac{1}{|x|} p_{Y}\left(\frac{z}{x}\right) .
$$

\section{References}

[1] G. Aubert and J.-F. Aujol. A variational approach to removing multiplicative noise. SIAM Journal on Applied Mathematics, 68(4):925-946, 2008.

[2] A. Buades, B. Coll, and J.-M. Morel. Online demo: Non-local means denoising. http: //www.ipol.im/pub/algo/bcm_non_local_means_denoising.

[3] A. Buades, B. Coll, and J.-M. Morel. A non-local algorithm for image denoising. In IEEE Computer Society Conference on Computer Vision and Pattern Recognition, volume 2, pages $60-65,2005$.

[4] A. Buades, B. Coll, and J.-M. Morel. Image denoising methods. A new nonlocal principle. SIAM Review, 52(1):113-147, 2010.

[5] P. Coupé, P. Hellier, C. Kervrann, and C. Barillot. Nonlocal means-based speckle filtering for ultrasound images. IEEE Transactions on Image Processing, 18(10):2221-2229, 2009.

[6] A. DasGupta. Fundamentals of Probability: A First Course. Springer Texts in Statistics. Springer, first edition, 2010.

[7] C.-A. Deledalle, L. Denis, and F. Tupin. Iterative weighted maximum likelihood denoising with probabilistic patch-based weights. IEEE Transactions on Image Processing, 18(12):2661-2672, 2009.

[8] C.-A. Deledalle, F. Tupin, and L. Denis. Poisson NL means: Unsupervised non local means for Poisson noise. In Proceedings of ICIP, 2010.

[9] L. Denis, F. Tupin, J. Darbon, and M. Sigelle. SAR image regularization with fast approximate discrete minimization. IEEE Transactions on Image Processing, 18(7):15881600, 2009.

[10] S. Durand, J. Fadili, and M. Nikolova. Multiplicative noise removal using L1 fidelity on frame coefficients. Journal of Mathematical Imaging and Vision, 36(3):201-226, 2010. 
[11] J. Fan, M. Farmen, and I. Gijbels. Local maximum likelihood estimation and inference. Journal of the Royal Statistical Society, Series B, Statistical methodology, pages 591-608, 1998.

[12] G. Gilboa, J. Darbon, S. Osher, and T. Chan. Nonlocal convex functionals for image regularization. UCLA CAM Report, 06-57, 2006.

[13] G. Gilboa and S. Osher. Nonlocal linear image regularization and supervised segmentation. Multiscale Modeling \& Simulation, 6(2):595-630, 2007.

[14] G. Gilboa and S. Osher. Nonlocal operators with applications to image processing. Multiscale Modeling ES Simulation, 7(3):1005-1028, 2008.

[15] G. R. Grimmett and D. R. Stirzaker. Probability and Random Processes. Oxford University Press, third edition, 2001.

[16] J. Jacod and P. Protter. Probability Essentials. Springer, second edition, 2004.

[17] A. Jeffrey and H.-H. Dai. Handbook of Mathematical Formulas and Integrals. Academic Press, fourth edition, 2008.

[18] C. Kervrann, J. Boulanger, and P. Coupé. Bayesian non-local means filter, image redundancy and adaptive dictionaries for noise removal. In F. Sgallari, A. Murli, and N. Paragios, editors, Scale Space and Variational Methods in Computer Vision, volume 4485 of $L N C S$, pages 520-532. Springer, 2007.

[19] Y. Matsushita and S. Lin. A probabilistic intensity similarity measure based on noise distributions. In IEEE Conference on Computer Vision and Pattern Recognition, CVPR '07, 2007.

[20] J. Polzehl and V. Spokoiny. Propagation-separation approach for local likelihood estimation. Probability Theory and Related Fields, 135(3):335-362, 2006.

[21] Quickbird sensor, Digital Globe. Optical image.

[22] V. K. Rohatgi. An Introduction to Probability Theory and Mathematical Statistics. John Wiley \& Sons, Inc., 1976.

[23] T. A. Severini. Likelihood Methods in Statistics, volume 22 of Oxford statistical science series. Oxford University Press, 2000.

[24] G. Steidl and T. Teuber. Removing multiplicative noise by Douglas-Rachford splitting methods. Journal of Mathematical Imaging and Vision, 36(2):168-184, 2010.

[25] N. Wiest-Daesslé, S. Prima, P. Coupé, S. P. Morrissey, and C. Barillot. Rician noise removal by non-local means filtering for low signal-to-noise ratio MRI: applications to DT-MRI. In D. Metaxas, L. Axel, G. Fichtinger, and G. Szekely, editors, Medical Image Computing and Computer-Assisted Intervention - MICCAI 2008, 11th International Conference, Proceedings, Part II, volume 5242 of LNCS, pages 171-179. Springer, 2008. 